\title{
Planejamento e desenho urbanos: uma conciliação possível?
}

\author{
Urban planning and design: a possible conciliation?
}

Danielle Pereira Montrezor[a] (D), Sidney Piochi Bernardini[a] (D)

[a] Universidade Estadual de Campinas (UNICAMP), Campinas, SP, Brasil

Como citar: Montrezor, D. P., \& Bernardini, S. P. (2019). Planejamento e desenho urbanos: uma conciliação possível?. urbe. Revista Brasileira de Gestão Urbana, 11, e20180133. https://doi.org/10.1590/2175-3369.011.e20180133

\section{Resumo}

As cidades vivem um momento de revalorização do desenho urbano, ainda que, em geral, continuem a enfrentar desafios transcendentes à constituição meramente física. Mas, então, o que está sendo pensado para tornar a cidade mais acolhedora e viva em relação ao seu desenho? A recente revisão do Plano Diretor Estratégico (PDE) e da Lei de Parcelamento, Uso e Ocupação do Solo (LPUOS) do município de São Paulo aparentemente converge para possíveis soluções abordadas na literatura do campo disciplinar do desenho urbano desde sua consolidação, na década de 1960. A pesquisa propõe elucidar, portanto, como se dá a relação entre a literatura científica e o que está sendo proposto por essas normas. Para isso, foram realizados estudos documentais das normas, revisão bibliográfica relacionada ao tema e ainda revisão sistemática da literatura (RSL) para o levantamento da produção científica no campo do desenho urbano. Os resultados revelam que há uma tendência de crescente valorização da escala do pedestre no processo de planejamento urbano, com ênfase na correlação entre uso do solo, mobilidade urbana e valorização da dimensão humana da cidade e que isso, de certa forma, reflete nas propostas apresentadas pelas normas recentemente instituídas em São Paulo.

Palavras-chave: Desenho urbano. Regulação urbana. Planejamento urbano. Revisão sistemática da literatura.

\section{Abstract}

The cities are living a moment of revaluation of the urban design, although in general, continuing to face the transcendence of its merely physical structure. Then, what is being thought to make the city more welcoming and alive in relation to its design? The recent review of the Strategic Master Plan and Land Use and Occupation Act of São Paulo municipality apparently converge to possible solutions that have been addressed by the literature related to the field of Urban Design since its consolidation in the '60s. Therefore, this research aims to elucidate the relationship between the literature and what is being proposed by these reviewed norms. For this purpose, documentary studies of the norm and a literature review related to the theme were carried out, and the method of Systematic Review of Literature (SRL) was applied to survey the scientific production related to urban design. Results have demonstrated a 
trend towards a growing appreciation of the pedestrian scale within the urban planning process, with emphasis on the correlation between land use, urban mobility and the city human dimension enhancement and these reflect - a certain extent in the presented urban regulation proposals.

Keywords: Urban design. Urban regulation. Urban planning. Systematic literature review.

\section{Introdução}

Grande parte da vida nas cidades acontece nos espaços públicos oferecidos, os quais seguem em constante transformação, fruto de processos históricos, sociais e econômicos, e podem ainda ser formados e transformados a partir de diretrizes do desenho urbano. Vive-se um momento de revalorização do desenho urbano, ainda que as cidades, em geral, continuem a enfrentar desafios que transcendem a sua constituição meramente física, porém ainda não há consenso sobre o que é mais adequado em relação à sua forma (Lamas, 2004). Mas, então, quais temas têm sido abordados dentro da temática do desenho urbano? 0 que está sendo pensado para tornar a cidade mais acolhedora e viva?

Conforme afirma Del Rio (1990), o desenho urbano, no âmbito internacional, consolidou-se como uma área de conhecimento interdisciplinar vinculada ao planejamento urbano na década de 1960, quando surgiram novas necessidades associadas à qualificação do espaço urbano e à adoção de medidas de controle do ambiente construído para torná-lo mais humano diante do contexto em que o conhecimento se expandia, de um lado, em uma perspectiva humanística das Ciências Sociais e, de outro, com um foco realista de áreas ligadas à economia e à educação. Ao lado dessas reflexões, há um conjunto de escritos que denuncia aspectos essenciais da vida urbana, inserindo críticas contundentes ao planejamento urbano que se praticava a partir do segundo pós-guerra. Nesse contexto, a produção literária, que passou a ser amplificada, foi decisiva para criar um ambiente de discussão que contribuiu para a consolidação do seu campo disciplinar. Autores, como Jane Jacobs, Kevin Lynch, Christopher Alexander, Robert Venturi, Colin Rowe, Edward Relph, além de outros, imprimiram suas marcas nesse processo de consolidação (Macdonald \& Larice, 2013). A essa lista é possível acrescentar outros nomes, como Gordon Cullen, Donald Appleyard, Rob e Leo Krier, Peter Calthorpe, que também tiveram seus escritos ou suas experiências, de certa forma, conhecidos no Brasil. Alguns desses autores tiveram uma ampla disseminação a partir da tradução de suas obras, o que explica, em certa medida, o curso da consolidação do campo disciplinar no país e as temáticas que se vincularam aos processos de formação da cidade, que passaram a ser estruturados nas escolas de arquitetura e urbanismo e ampliados no campo profissional. Mais recentemente, outros autores ganharam notoriedade a partir da tradução de suas obras, evidenciando aspectos que retomam a importância da reativação da vida nas cidades e a reorientação quanto aos princípios de sustentabilidade: Jan Gehl (2015), Jeff Speck (2016) e Douglas Farr (2013).

O desenho urbano integra um amplo processo no âmbito do planejamento territorial entre a escala do planejamento urbano e do edifício, exatamente onde há uma falha no processo de construção da cidade, conforme aponta Noto (2017). As normas que regulam o uso e a ocupação do solo foram fundamentadas, historicamente, por indicadores e parâmetros que vislumbravam o alcance de um equilíbrio ideal nas relações de causas e efeitos, a partir de regras limitadas em relação ao desenho urbano (recuos, taxa de ocupação, coeficiente de aproveitamento e taxa de permeabilidade). Essas regras, em sua maioria, não estimulam a densificação ou a diversificação funcional no meio urbano (Moraes \& Saboya, 2010) e reforçam a aplicação embasada em alocações uniformes bidimensionais de uso do solo, distanciando-as de questões de qualificação do espaço urbano a partir de conceitos do desenho urbano (Hall, 2000).

A geometria "homogênea" do desenho, pautada no zoneamento tradicional, diverge dos aspectos apontados como essenciais para a vida urbana, por exemplo, diversidade de tipologias de edifícios e de usos, traçado viário bem conectado, elementos visuais que permitam a legibilidade da cidade, valorização do pedestre, entre outros fatores, que ajudam a garantir a dinâmica necessária para a 
integração da sociedade no espaço público (Mehta, 2009; Cervero, 2009; Hall, 2008). 0 contexto nacional na década de 1960, que precedeu à fase de consolidação do campo disciplinar no Brasil, limitou o debate político acerca do planejamento urbano e estabeleceu um padrão tecnicista nas normas que passaram a regular a produção da cidade, culminando na consolidação do zoneamento como ferramenta reguladora do uso e da ocupação do solo (Nobre, 2006). Sendo assim, a possibilidade de inovação desse instrumento de regulação pode ser de grande valor para a superação de desafios decorrentes da complexidade do processo de urbanização das cidades brasileiras.

O modelo tradicional da legislação urbana tem sido discutido por muitos autores em um contexto de avanços em relação à produção do espaço público qualificado que demanda atitudes menos reducionistas na formulação e na gestão do zoneamento. Punter (2007) observa que o instrumento do zoneamento poderia ser adaptado para se tornar mais flexível e mais sensível ao desenho. 0 autor destaca que, nessa busca de sinergia entre planejamento e desenho, seria importante aproveitar, mais largamente, o alcance dos atores e dos instrumentos administrativos (taxas, subsídios e aquisição de terras), além de mitigar os efeitos excludentes das estratégias de controle e de regulação do desenho sob maior integração entre o planejamento e o zoneamento, ponderando as limitações do segundo. Considerando que os aspectos restritos ao controle e seus mecanismos de gestão não conseguem acomodar a espontaneidade orgânica, a vitalidade, a inovação e o pluralismo, o instrumento poderia se tornar menos prescritivo e mais instrutivo. Hall (2008), por sua vez, considera as limitações restritas à lógica do uso do solo insuficientes, defendendo que a forma construída permanece por muito mais tempo que os usos instalados, e, nesse sentido, uma atenção maior deveria ser dada ao conjunto morfológico estabelecido. Para além do atendimento restrito aos códigos, a concepção de novos núcleos urbanos deveria incorporar melhor os processos de projeto, abarcando a discussão, no campo da gestão pública, sobre os aspectos físico-morfológicos desde o início da concepção, incluindo o formato e o desenho das quadras, a relação dos edifícios com as ruas e a permeabilidade do conjunto urbano em relação ao contexto em que será inserido.

Outro aspecto mencionado pela literatura diz respeito às estratégias de caminhabilidade e à interação nos espaços públicos, especialmente os passeios públicos e sua relação com o uso do solo - questão recorrente que perpassa as preocupações nas formulações dos códigos de zoneamento. Mehta (2009), por exemplo, conseguiu identificar, a partir da investigação em três áreas na cidade de Somerville, Região Metropolitana de Boston, os atributos que tornavam as experiências dos pedestres mais interessantes, duradouras e potencializadoras de interações sociais. Tais atributos vão além, muitas vezes, da simples relação com o uso comercial dos edifícios lindeiros e correspondem à qualidade espacial dos próprios passeios, suas configurações, suas dimensões e os elementos que os constituem. Essa questão é complementada com as preocupações referentes aos motivos pelas quais as pessoas escolhem alguns meios de deslocamento em detrimento de outros e à maneira como a qualidade espacial pode interferir nessas escolhas. Comunidades compactas, usos mistos e desenhos de vias que valorizem modos de transporte alternativos podem influenciar diretamente a escolha das pessoas em relação ao tipo de transporte.

A recente revisão do Plano Diretor Estratégico (PDE) do município de São Paulo (Lei no 16.050/14) (São Paulo, 2014) aparenta trilhar esse debate ao inserir novas diretrizes de uso e ocupação do solo potencializadoras da escala do desenho urbano. A sequência desse plano foi a aprovação, em 2016, da Lei de Parcelamento, Uso e Ocupação do Solo (LPUOS) do município (Lei no 16.402/16) (São Paulo, 2016), que abarcou algumas dessas proposições. Conforme colocado em seu material de divulgação, a revisão do PDE foi realizada a partir da compreensão profunda do território urbano, admitindo conceitos e mecanismos capazes de corroborar o processo de superação de desafios relacionados à mobilidade urbana, à qualidade da vida nos bairros, à orientação do crescimento urbano, entre outros aspectos competentes às dinâmicas do processo de transformação urbana de uma metrópole (São Paulo, 2014).

Ao longo do processo de revisão do PDE (2014), as audiências públicas para a discussão do projeto de lei contaram com a participação de professores e pesquisadores universitários, os quais trouxeram 
em pauta a discussão de importantes temas relacionados a questões sociais, ambientais e de mobilidade do âmbito do desenho urbano. Em continuidade ao processo, na revisão da LPUOS (2016) foram realizados o Concurso Nacional Ensaios Urbanos: Desenhos para o Zoneamento de São Paulo e o Atelier Ensaios Urbanos, dos quais diversos cursos de arquitetura e urbanismo participaram, a partir da necessidade de compreender tridimensionalmente as propostas referentes ao uso e à ocupação do solo já sinalizadas no PDE. Esse envolvimento da academia na revisão do plano e seus instrumentos pode representar uma sinergia entre os aspectos discutidos na produção científica e as propostas revistas para regulação de uso e ocupação do solo no município de São Paulo, possibilitando a abertura de um canal para a incorporação dos achados científicos mais recentes, no campo do desenho urbano, na construção do marco urbanístico paulistano.

O novo PDE parece, assim, convergir para respostas de questões enfrentadas pelo município, que, historicamente, insiste no planejamento que valoriza o transporte individual motorizado em detrimento dos demais meios, além de não apontar soluções eficazes para questões dos fluxos do transporte viário, da qualidade ambiental e da qualidade do transporte público (Lajut, 2016). Considerando esse panorama, o objetivo do artigo é compreender a relação entre conceitos e parâmetros estabelecidos no PDE (Lei no 16.050/14) (São Paulo, 2014) e na LPUOS (Lei no 16.402/16) (São Paulo, 2016) de São Paulo e os conhecimentos difundidos acerca do desenho urbano, observando em que medida avanços nas pesquisas dessa área aparecem nesse conjunto de instrumentos, reunindo a produção científica que correlaciona desenho urbano e planejamento urbano por meio do método de revisão sistemática da literatura (RSL). A pesquisa propõe elucidar, assim, ideias que culminaram na formação do cenário atual - no caso, o atual PDE e a LPUOS. Para tanto, a estrutura do artigo - após introduzir o tema e sua problemática - é dividida em três partes: a primeira apresenta o método com foco na explicação das etapas da RSL; posteriormente, são apresentados os resultados da RSL; e, na terceira, há a comparação entre esses resultados e os conteúdos sistematizados do PDE (2014) e da LPUOS (2016)).

\section{Método}

Os procedimentos metodológicos partem da relação entre duas variáveis: de um lado, o conjunto teórico-conceitual acerca do campo disciplinar do desenho urbano contemporâneo e, de outro, os conceitos e as diretrizes estabelecidos no PDE (Lei no 16.050/14) e na LPUOS (Lei no 16.402/16) de São Paulo. Foi realizado, primeiramente, o estudo do referencial documental e bibliográfico relacionado ao planejamento urbano, à regulação do uso e da ocupação do solo e ao desenho urbano em documentos nacionais e internacionais primários e secundários, incluindo livros e artigos científicos. Posteriormente, foi realizado o estudo dos instrumentos legais instituídos: o PDE (Lei no 16.050/14) e a LPUOS (Lei no 16.402/16) do município de São Paulo.

Com o objetivo de sistematizar dados relacionados à produção acadêmica que envolve a temática do desenho urbano, foi utilizado o método de revisão sistemática de literatura (RSL). A RSL é um estudo secundário por meio do qual pesquisas disponíveis em estudos primários de relevância são identificadas (Kitchenham et al., 2009). Esse método permite que, a partir de uma problemática inicial, seja feita a obtenção de resultados pertinentes identificando conteúdos da produção científica de forma criteriosa (Brereton et al., 2007). Após a realização dos procedimentos metodológicos que fazem parte da RSL, representados na Figura 1, os dados obtidos foram sistematizados, gerando resultados quantitativos (bibliométricos) e qualitativos. Conforme aponta Vanti (2002), a bibliometria é um método eficaz de mapeamento da estrutura do conhecimento em relação a determinado campo, possibilitada pela pesquisa quantitativa dos dados bibliográficos, a partir do uso de palavras-chave, a fim de avaliar as tendências ligadas ao tema de estudo. A RSL se deu a partir da identificação das produções científicas que fazem a interface entre desenho urbano e planejamento urbano. 
As bases de dados para a RSL foram selecionadas pelo sistema de busca no Portal de Periódicos da CAPES ${ }^{1}$, a partir da área e da subárea de conhecimento "Ciências Sociais Aplicadas" e "Planejamento Urbano e Regional", respectivamente. 0 resultado apresentou 43 bases, das quais 7 foram selecionadas, de acordo com os seguintes critérios: possuir textos completos; ter sistemas de busca semelhantes, permitindo a busca de string no resumo do artigo, uma vez que a busca nas palavras-chave se mostrou menos abrangente; gerar resultado substancial com a busca. A string de busca definida foi "urban design" AND ("public space" OR "urban planning" OR "zoning" OR "urban parameter" OR "land use" OR "urban legislation"). A busca se limitou à presença de strings de busca nos resumos de artigos em periódicos científicos. Supondo que a maior parte das pesquisas científicas gera artigos ao longo de seu desenvolvimento, esse foi considerado o meio de divulgação mais completo para se chegar ao resultado esperado no processo de revisão. Os artigos também foram selecionados pelo idioma no qual seu texto completo foi escrito (Filtro 1), aplicado durante a busca nas bases, sendo mantidos aqueles em português, inglês e espanhol, visando à possibilidade da leitura do artigo completo. Os softwares utilizados no processo de organização dos dados dos artigos coletados foram Zotero e Mendley. Por fim, foi realizada a leitura dos títulos e, na sequência, dos resumos, bem como eventuais leituras dos textos completos para a seleção dos artigos aderentes à pesquisa.

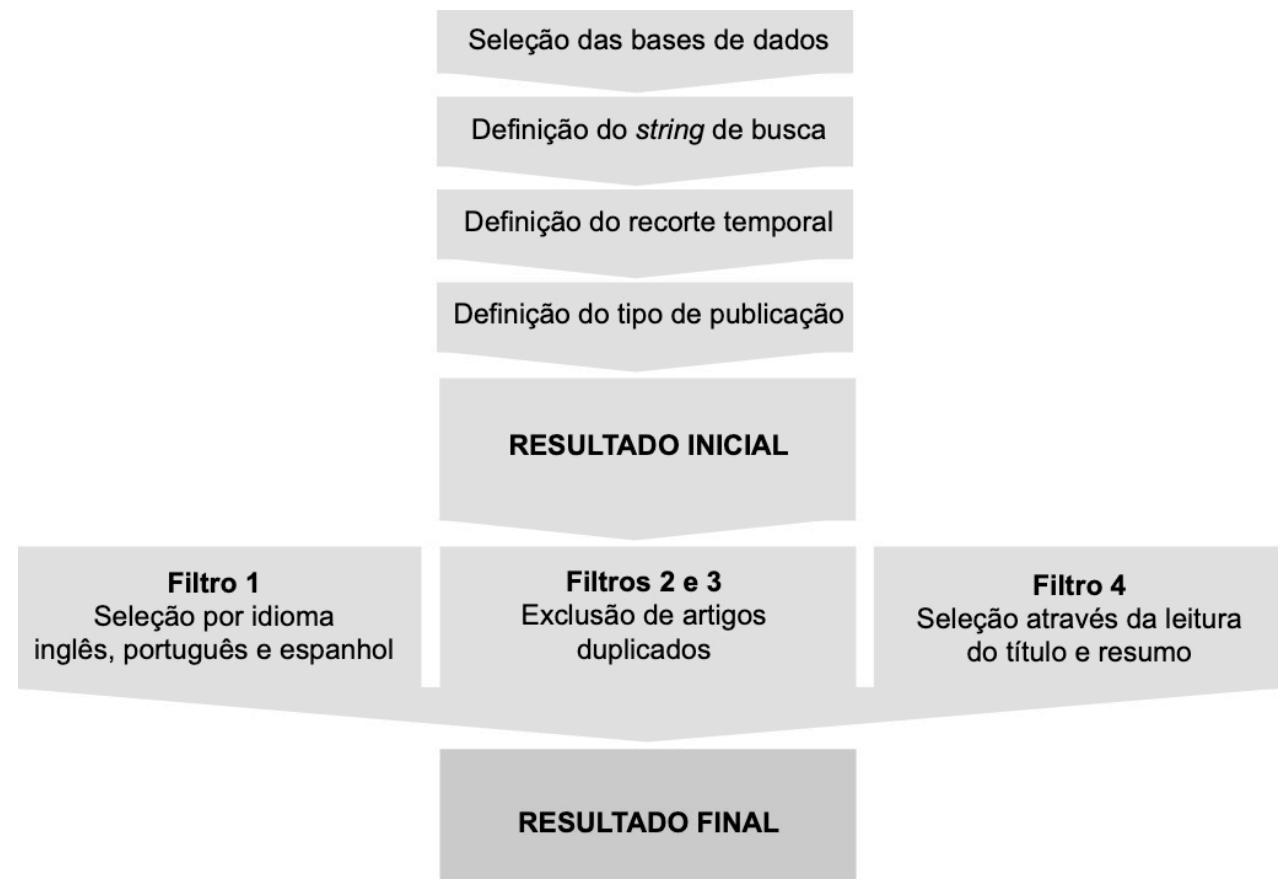

Figura 1 - Procedimentos realizados na revisão sistemática de literatura. Fonte: elaboração dos autores (2017).

Outra importante etapa da RSL foi a delimitação do recorte temporal, estabelecido no intervalo entre os anos de 1996 e 2017. Adotou-se como marco inicial a primeira edição da "Carta do Novo Urbanismo" (The Charter of the New Urbanism), publicada em 1996 e apresentada no IV Congresso para o Novo Urbanismo Norte-Americano, que traz princípios relacionados ao planejamento e ao desenho urbano. Seus 27 princípios abrangem desde a escala regional, passando pela escala do bairro, até a escala da quadra, da rua e das edificações. A Carta tem como objetivos evitar o crescimento urbano disperso, promover a acessibilidade por meio de transporte coletivo, incentivar a criação de comunidades compactas e estimular a participação social no processo de planejamento urbano (Macedo, 2007).

Após a seleção inicial, foi realizada a extração das palavras-chave presentes nos artigos com o objetivo de analisar qualitativamente os temas abordados. Nessa etapa, foi constatado que alguns não possuíam

\footnotetext{
10 acesso ao Portal, assim como às bases de dados, foi realizado a partir da conexão oferecida pelo servidor da Universidade à qual os autores são filiados (CAPES, 2018).
} 
palavras-chave, as quais, nesses casos, foram atribuídas a partir das leituras dos resumos ou do artigo completo. Isso foi recorrente em quase todas as bases de dados, com exceção do SciELO e da Emerald. Após esse procedimento, todas as palavras foram compiladas em um único arquivo em formato.docx e contabilizadas com o auxílio de uma ferramenta de análise de texto ${ }^{2}$ disponibilizada on-line. Essa ferramenta contabiliza as palavras que aparecem com mais recorrência em um texto. Com o objetivo de tornar o resultado coerente com o material identificado, foram desconsiderados os termos referentes a nomes próprios, como nome de países, e somadas palavras-chave iguais que apareceram no singular e no plural, por exemplo "neighborhood" e "neighborhoods", que foram contabilizadas juntas.

\section{A interface entre desenho urbano e planejamento urbano}

A interface entre o campo disciplinar do desenho urbano e o planejamento urbano tem sido crescente entre os estudos do meio urbano, uma vez que as estratégias de planejamento e desenho urbanos articuladas podem contribuir para reorganizar os espaços urbanos e transformar os comportamentos das pessoas (Wey, 2011), promovendo qualidade de vida na cidade. Na Tabela 1, estão os resultados quantitativos, sendo necessário esclarecer que o Filtro 3 eliminou artigos duplicados entre as bases. No Filtro 4, foram realizadas as leituras dos títulos e dos resumos dos artigos, com o objetivo de excluir textos que não estivessem no formato de artigo e aqueles que não tivessem uma abordagem diretamente vinculada à temática do desenho urbano, mas que, apesar disso, continham os termos da string de busca e que, portanto, apareceram na busca.

Tabela 1 - Dados quantitativos obtidos com a RSL

\begin{tabular}{cc}
\hline Base de dados & Resultado inicial \\
\hline ASP & 250 \\
Emerald & 11 \\
JSTOR & 24 \\
Sage & 13 \\
SciELO & 78 \\
Scopus & 491 \\
SocINDEX & 36 \\
Total inicial & 903 \\
Filtro 2 - Duplicidade & 732 \\
Filtro 3 - Conteúdo & 717 \\
Resultado final & 717 \\
\hline
\end{tabular}

Fonte: elaboração dos autores com base nos dados da RSL (2018).

Na Figura 2, é notável o desequilíbrio entre a quantidade de artigos totais e nacionais nos periódicos indexados nas bases de busca ${ }^{3}$. Foram considerados artigos nacionais os escritos por autores vinculados a instituições brasileiras. Os 23 artigos brasileiros coletados abordam temas variados. A qualidade ambiental é o principal tema, foco das pesquisas que abordam o desenho urbano sob a perspectiva de mitigar as alterações climáticas nas cidades e garantir o conforto térmico (SiqueiraGay et al., 2017; Krüger et al., 2015; Monteiro et al., 2012; Barros et al., 2010), a gestão de água atrelada ao desenho urbano (Nunes et al., 2011; Vasconcelos et al., 2016; Silva \& Souza, 2016), a importância da arborização urbana (Gonçalves \& Meneguetti, 2015) e a qualidade da ventilação natural nos espaços urbanos (Tork et al., 2017). A questão da habitação de interesse social (HIS) é o segundo principal tema entre as publicações nacionais, apresentada com o direito à cidade (Budds \& Teixeira, 2005) e em relação às normas municipais referentes à HIS (Silva, 2011).

\footnotetext{
${ }^{2}$ A ferramenta de análise de texto utilizada está disponível em Online-Utility.org (2018).

${ }^{3} \mathrm{~A}$ pequena quantidade de artigos pode representar uma possível falta de publicação nacional em revistas internacionais, o que leva à concentração de conhecimento, e prejudicar os ganhos que a difusão deles pode gerar.
} 


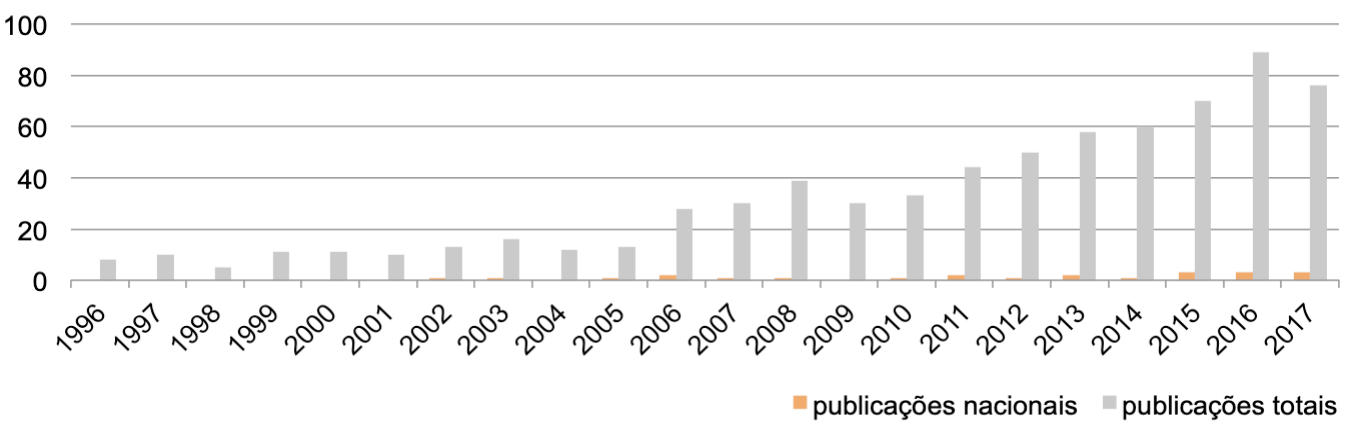

Figura 2 - Quantidade de artigos ao longo dos anos. Fonte: elaboração dos autores com base nos dados da RSL (2018).

A compreensão do cenário das pesquisas realizadas que associam os termos buscados é relevante para entender a atual produção científica com enfoque no processo de materialização do desenho urbano nas cidades - no caso, o planejamento urbano, a legislação urbana, o zoneamento, o uso do solo e os parâmetros urbanos. Após a seleção, com o objetivo de compreender de forma direta os temas abordados pelos artigos, foram levantadas suas palavras-chave. As 55 palavras-chave mais presentes entre os artigos (Figura 3) correspondem a 30\% do total delas e a 1.227 ocorrências.

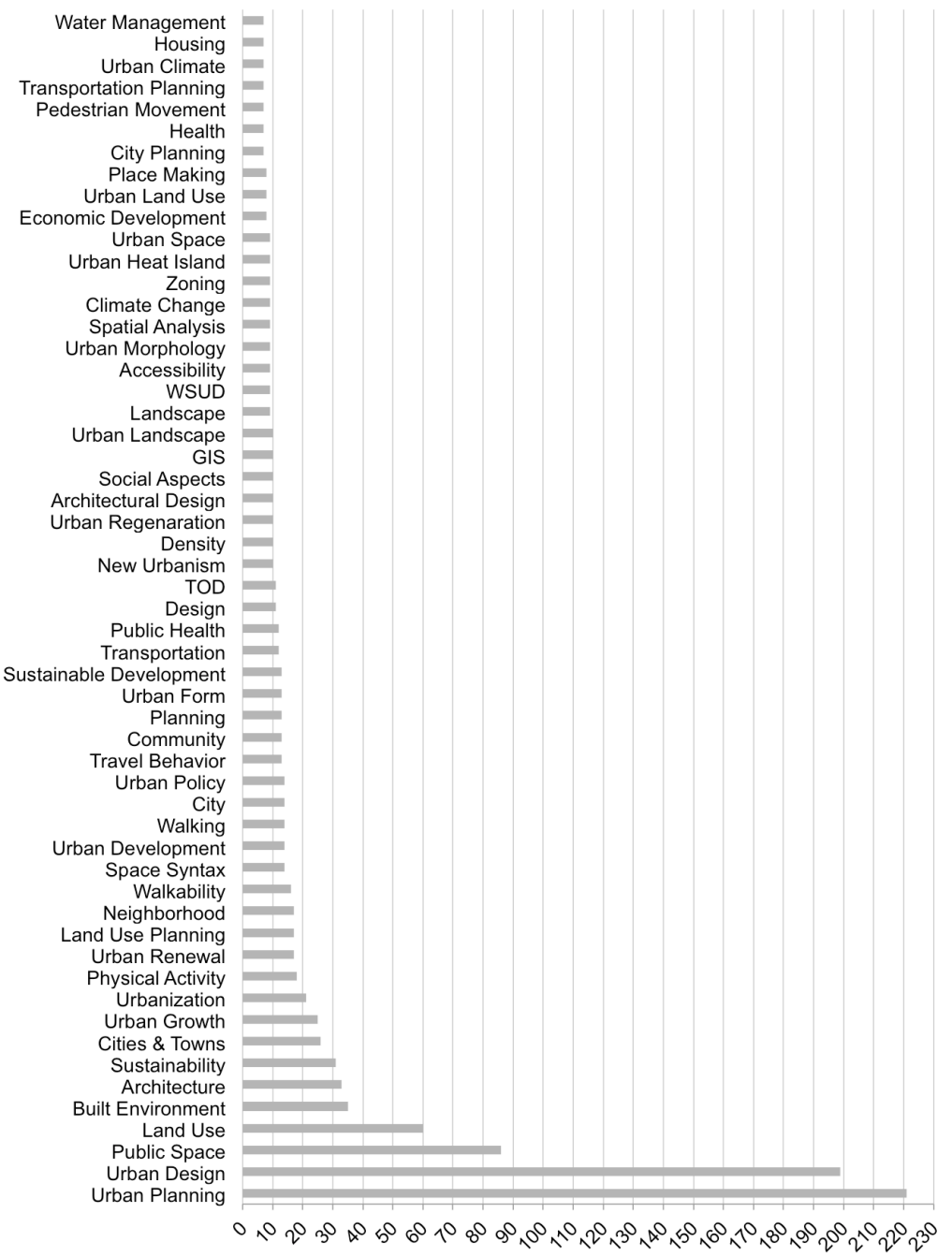

Figura 3 - Palavras-chave mais utilizadas nos artigos. Fonte: elaboração dos autores com base nas palavras-chave extraídas dos artigos (2018). 
Entre as principais palavras-chave, nove tem relação direta com o estudo do ambiente construído: "urban design", "built environment", "space syntax", "urban form", "architecture", "architecture design", "spatial analysis", "density" e "urban morphology". Uma vez que a pesquisa correlaciona desenho urbano e termos associados à sua materialização no meio urbano, é compreensível que o ambiente edificado seja recorrente nas pesquisas.

Termos relacionados ao processo do planejamento urbano, bem como alguns de seus instrumentos, também foram recorrentes, aparecendo em quantidades significativas: "urban planning", "land use", "land use planning", "city planning", "zoning", "urban growth", "urbanizatoin", "planning", "urban renewal", "urban regeneration", "urban development", "sustainable development" e "urban land use". As palavras-chave "climate change", "urban climate", "urban heat-island", "water management" e "Water Sensitive Urban Design (WSUD)" também estão nessa lista e correspondem a temáticas relacionadas à qualidade do meio ambiente. Conforme aponta Farr (2013), trata-se de uma temática essencial para o urbanismo, tendo em vista que, com o passar do tempo, o urbano se alienou do meio ambiente, e esse processo deve ser revertido integrando princípios ecológicos às cidades e ao estilo de vida urbano.

Outro tema em destaque é a mobilidade urbana, representada pelas palavras-chave "travel behaviour", "transit-oriented development (TOD)", "transportation planning" e "transportation". 0 termo TOD, traduzido para Desenvolvimento Orientado pelo Transporte Público (DOT), faz parte das novas abordagens em relação ao desenho urbano e provém da necessidade crescente de maior acessibilidade, gerando empreendimentos que melhorem a relação entre moradores, transporte público e meios alternativos de mobilidade (Farr, 2013). Em complemento a esses termos, há o destaque dos termos relacionados à escala do pedestre, convergindo para o conceito de caminhabilidade (Speck, 2016), representado pelas palavras "walkability", "walking", "pedestrian movement" e "travel behavior". Faz-se necessário ainda ressaltar as palavras-chave "physical activity", "public health" e "health", diretamente relacionadas à saúde, ao bem-estar e ao transporte ativo, questões colocadas como um dos principais argumentos para tornar as cidades mais caminháveis (Speck, 2016) e promover meios de deslocamentos não motorizados.

\section{Desenho urbano e qualidade dos espaços públicos}

Após a leitura dos artigos, foram selecionados 204, aderentes a questões diretamente ligadas ao desenho urbano como qualificador do espaço público (Apêndice A). Esses artigos foram categorizados pelos assuntos abordados (Tabela 2). 0 tratamento dos espaços públicos foi tomado como referência para a seleção desses artigos, uma vez que é onde o desenho urbano tem maior potencial de atuação para melhoria da qualidade do espaço urbano e, consequentemente, da qualidade de vida das pessoas, visto que esses espaços promovem as interações sociais e culturais (Carmona et al., 2003).

O objetivo dessa seleção é compreender o que tem sido abordado pelas pesquisas referentes à interface entre desenho urbano e planejamento urbano em relação aos espaços públicos da cidade, considerados como todos aqueles espaços livres de acesso público, como vias, parques etc. A Tabela 2 contempla todos os assuntos tratados pelos artigos aderentes a essa seleção, bem como a quantidade de artigos que correspondem a cada um deles. Os temas estão identificados por meio de um ID correspondente ao artigo no quadro em anexo (Apêndice A). Essa identificação se deu por meio da aproximação entre os assuntos para facilitar a compreensão e a visualização da natureza dos temas abordados, sendo definidas quatro categorias: (1) valorização da escala humana nos espaços urbanos; (2) melhoria na infraestrutura de transporte; (3) busca do equilíbrio ambiental; e (4) temas que correlacionam todas as três categorias. 
Tabela 2 - Assuntos abordados pelos artigos selecionados

\begin{tabular}{|c|c|c|}
\hline ID & Assuntos abordados pelos artigos & $\begin{array}{c}\text { Quantidade de } \\
\text { artigos }\end{array}$ \\
\hline A & Relação entre as características do espaço físico e o uso de transportes não motorizados & 51 \\
\hline B & Qualificação de espaços públicos e vitalidade urbana por meio do desenho urbano & 17 \\
\hline C & Desenho urbano como promotor de segurança & 10 \\
\hline D & Cultura, identidade e percepção das pessoas sobre os espaços urbanos & 9 \\
\hline $\mathrm{E}$ & Impactos sociais do desenho urbano & 5 \\
\hline $\mathrm{F}$ & Arte urbana como elemento qualificador do espaço público & 4 \\
\hline G & Necessidades dos idosos no desenho urbano & 3 \\
\hline $\mathrm{H}$ & Relação entre desenho urbano e diversidade nas cidades & 3 \\
\hline 1 & Senso de comunidade e desenho urbano & 2 \\
\hline $\mathrm{J}$ & Relação entre o perfil socioeconômico e a escolha do modo de transporte & 1 \\
\hline K & Qualificação da faixa edificada um nível acima da escala do pedestre & 1 \\
\hline $\mathrm{L}$ & Implantação de edifícios catalíticos para promover fluxo de pedestres & 1 \\
\hline M & Importância do perímetro da quadra para a qualidade do espaço urbano & 1 \\
\hline A & Tratamento do desenho urbano em áreas de infraestrutura de transporte & 15 \\
\hline $\mathrm{B}$ & Soluções de desenho urbano para vagas de estacionamento & 5 \\
\hline $\mathrm{C}$ & Relação entre traçado viário, desenho urbano e desenvolvimento urbano & 2 \\
\hline $\mathrm{D}$ & Corredores de ônibus como solução para transporte público & 1 \\
\hline A & Condições climáticas nos espaços públicos e desenho urbano & 17 \\
\hline B & Gestão da água integrada ao desenho urbano & 8 \\
\hline $\mathrm{C}$ & Desenho urbano e ecossistema urbano & 6 \\
\hline $\mathrm{D}$ & Estratégias de intervenção urbana para reversão de ilhas de calor & 4 \\
\hline $\mathrm{E}$ & Importância dos parques urbanos para o equilíbrio ambiental & 3 \\
\hline $\mathrm{F}$ & Forma urbana e ventilação natural & 2 \\
\hline G & Qualidade do ar nos espaços públicos & 1 \\
\hline $\mathrm{H}$ & Importância da vegetação urbana & 1 \\
\hline A & Zoneamento como qualificador do desenho urbano & 7 \\
\hline $\mathrm{B}$ & Sustentabilidade no meio urbano a partir do desenho urbano & 6 \\
\hline C & Relação entre as transformações no desenho urbano e os planos urbanísticos & 5 \\
\hline $\mathrm{D}$ & Princípios gerais para boas práticas de desenho urbano & 6 \\
\hline $\mathrm{E}$ & Crescimento compacto e desenho urbano & 3 \\
\hline $\mathrm{F}$ & Adensamento e qualidade do ambiente urbano & 2 \\
\hline G & Desenho urbano monumental e paisagem urbana & 1 \\
\hline $\mathrm{H}$ & Novo urbanismo e desenho urbano & 1 \\
\hline \multicolumn{3}{|c|}{ Legenda: } \\
\hline A-M & Valorização da escala humana nos espaços urbanos. & \\
\hline A-D & Melhoria na infraestrutura de transporte. & \\
\hline $\mathrm{A}-\mathrm{H}$ & Busca do equilíbrio ambiental. & \\
\hline $\mathrm{A}-\mathrm{H}$ & Valorização da escala humana nos espaços urbanos e melhoria na infraestrutura de trans & oorte. \\
\hline
\end{tabular}

Fonte: elaboração dos autores com base nas análises da RSL (2018).

Ao sistematizar os temas de acordo com a escala a que se refere cada um (Figura 4), é possível observar que há predominância de abordagens na escala do pedestre, o que corresponde ao estudo do espaço edificado de seu entorno imediato e de como é possível promover espaços amigáveis ao pedestre por meio do desenho urbano. 


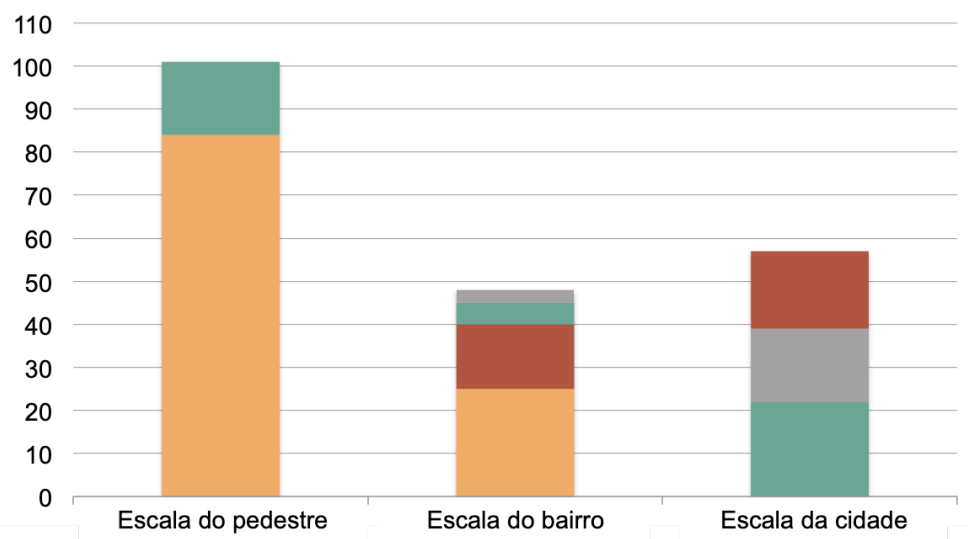

Figura 4 - Escala de abrangência dos temas. Fonte: elaboração dos autores com base nas análises da RSL (2018).

A partir dessa análise, é possível destacar os assuntos mais recorrentes (que estão entre os temas da Tabela 2), conforme apresentado na Figura 5, a qual indica os principais assuntos e a porcentagem de artigos. A presença de estudos que correlacionam desenho urbano com transporte é bastante forte, sendo base para os seguintes assuntos: (1) relação entre as características do espaço físico e o uso de transportes não motorizados, (2) tratamento de áreas próximas às estações de transporte (DOT) e (7) soluções de desenho urbano para vagas de estacionamento. 0 tema mais recorrente (1) aponta, em sua maioria, a contribuição da densidade, do uso misto e da conectividade das vias para cidades com maior fluxo de pedestres, e de meios de deslocamento não motorizados, como a caminhada e a bicicleta (Boulange et al., 2017; Lee et al., 2017; Gunn et al., 2017; Lee, 2015; Lerman \& Omer, 2016; Koohsari et al., 2016; Choi, 2014; Johnson \& Marko, 2008; Krizek, 2003; Greenwald, 2003; Handy et al., 2002).

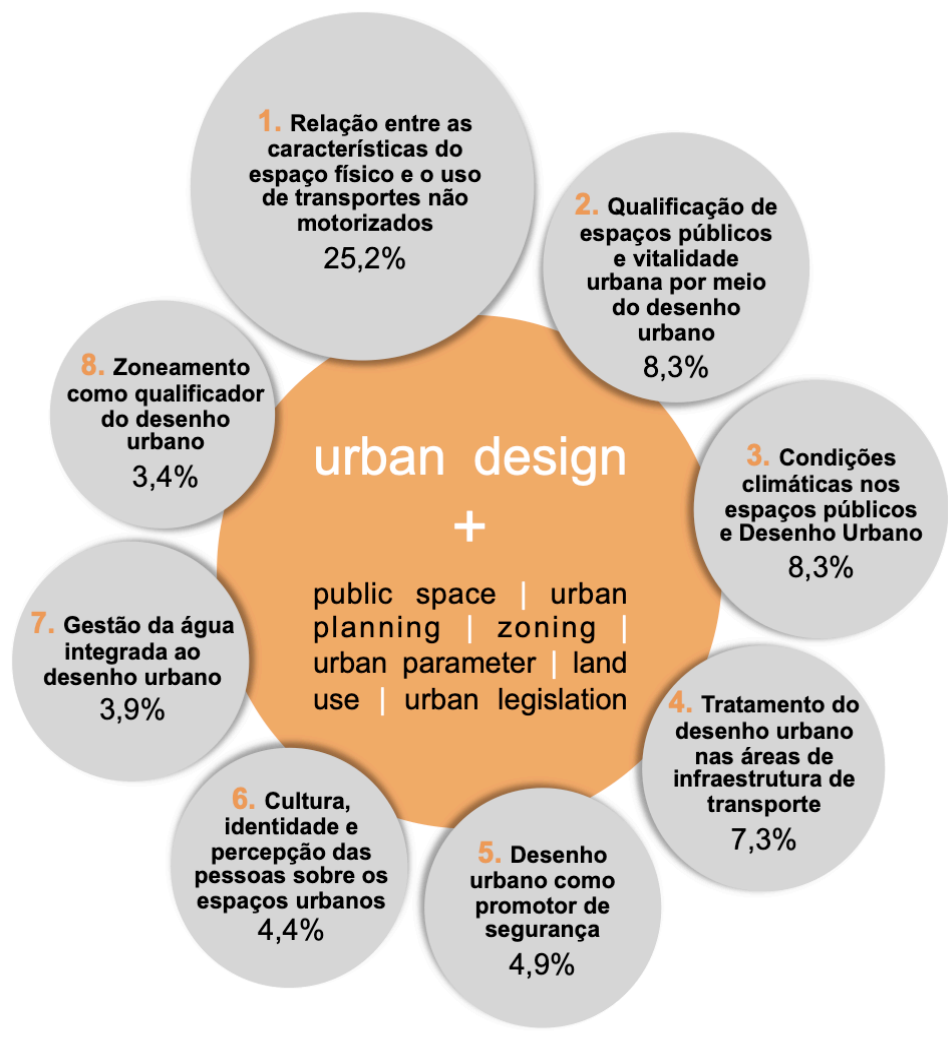

Figura 5 - Assuntos mais abordados e porcentagem de artigos. Fonte: elaboração dos autores com base nas análises da RSL (2018). 
Dentro dessa temática, há ainda artigos que trazem perspectivas complementares sobre a afirmação predominante. 0 uso misto, apesar de apontado pela maioria como importante estratégia para a qualificação de espaços urbanos, possui ressalvas, como a necessidade do estudo do tipo de mescla de usos e em qual intensidade ele deve ser incentivado (Dovey \& Pafka, 2017), uma vez que certos usos podem inibir o senso de comunidade que é essencial para o fluxo de pedestres (Wood et al., 2010). A densidade também é questionada como forma de promover espaços mais caminháveis, podendo não ser um conceito de desenho urbano saudável (Kemperman \& Timmermans, 2009). Além disso, projetos urbanos compactos podem não reduzir as viagens de deslocamento (Maat et al., 2005). A influência do espaço edificado, de forma geral, também é questionada, uma vez que varia de acordo com a finalidade dos deslocamentos. Viagens a trabalho são pouco influenciadas pela caminhabilidade dos espaços (Lee, 2015) e aspectos estéticos têm menor interferência no fluxo de pedestres do que infraestrutura do sistema viário, como estratégias de acalmamento de tráfego (Boarnet et al., 2011). Ainda, de forma mais ampla, verifica-se que todas as viagens programadas com um destino predefinido podem não ser influenciadas pela qualidade do trajeto (Koohsari et al., 2013).

Apesar de os termos predominantes se enquadrarem na categoria referente à valorização da escala humana nos espaços urbanos, há temas em destaque que abordam as demais categorias. 0 desenho urbano como ferramenta para alcançar o equilíbrio ambiental é explorado por dois temas mais presentes: (3) condições climáticas nos espaços públicos e desenho urbano e (7) gestão da água integrada ao desenho urbano (WSDU). 0 zoneamento como instrumento indutor do desenho urbano também aparece como um dos principais tópicos (8), o que reforça a atual discussão desse instrumento no planejamento urbano atual.

\section{A produção científica e as normas de regulação do solo}

A partir da nova Constituição Federal de 1988 e, posteriormente, com o Estatuto da Cidade (Brasil, 2001), o plano diretor se tornou obrigatório em municípios com mais de 20 mil habitantes, prevendo o uso do zoneamento como parte da estratégia de planejamento urbano e traçando diretrizes para embasar sua formulação. Sua revisão ocorre de 10 em 10 anos e também de acordo com o Estatuto da Cidade, o que deve se dar por meio de processo participativo. 0 município de São Paulo passou recentemente por esse processo ao aprovar a revisão do PDE e da LPUOS. Esse novo PDE tem como principal objetivo, de acordo com seu material de divulgação, promover a qualidade de vida para todos, diminuir o potencial construtivo em áreas consolidadas, estimular o crescimento da cidade ao longo de eixos de transporte público coletivo a partir do uso misto, gerar empregos e propiciar a vida social nos espaços públicos (São Paulo, 2014).

As estratégias para alcançar esses objetivos incluem, entre outros, a incorporação da agenda ambiental no desenvolvimento da cidade; a qualificação da vida urbana nos bairros; o fortalecimento da participação popular nas decisões dos rumos da cidade; o crescimento orientado nas proximidades do transporte público; a melhoria da mobilidade urbana; a socialização dos ganhos da produção da cidade (São Paulo, 2014). A LPUOS foi ainda mais objetiva nas diretrizes vinculadas ao desenho urbano, pautando-as na qualidade ambiental, na qualidade do desenho e da forma urbana, no fortalecimento da dimensão social, no melhoramento da gestão de impactos urbanísticos, no equilíbrio entre oferta de emprego e moradia, na preservação do patrimônio cultural e na simplificação da aplicação da lei.

Na Tabela 3, é possível diferenciar onde cada medida está presente e se consta no PDE, na LPUOS ou nos dois. No caso das medidas que estão presentes em ambos, estão dois importantes atributos de qualificação do espaço público, com o objetivo promover a vida urbana: a fachada ativa e a área de fruição pública. A fachada ativa é a ocupação do térreo localizado no alinhamento dos passeios por usos que não sejam residenciais, com acesso aberto para o logradouro público (São Paulo, 2014). A área de fruição pública, por sua vez, é uma área do pavimento térreo que deve ser de acesso público, sem nenhum tipo de fechamento ou vedação, podendo ter controle de acesso no período noturno (São Paulo, 
2016). Esses atributos visam promover o fluxo nos passeios públicos a partir do uso misto, aumentando o fluxo de pedestres por meio das qualidades do ambiente construído, e convergem para o principal assunto abordado pelos artigos estudados: a relação entre o meio construído e as escolhas das pessoas pelo tipo de transporte e ainda a relação entre uso misto, densidade e conectividade das vias com a caminhabilidade.

Tabela 3 - Propostas das Leis $n^{\circ} 16.050 / 14$ e $n^{\circ} 16.402 / 16$

\section{Propostas relacionadas ao desenho urbano - PDE (2014) e LPUOS (2016)}

Definição da largura mínima das vias

Alargamento, qualificação e manutenção de calçadas em atendimento às normas de acessibilidade universal

Nas áreas de influência dos eixos, nos lotes com frente para os eixos, o passeio deverá ter largura mínima de $5 \mathrm{~m}$

Desenvolvimento de projetos de hidrovias de forma integrada à requalificação da orla dos canais, das represas e dos lagos navegáveis,

transformando-os em espaços de convivência e embarque de passageiros e/ou portos de carga

Integração entre os sistemas de transporte coletivo, viário, cicloviário, hidroviário e de circulação de pedestres, dotando-os de condições adequadas de acessibilidade universal e sinalizações adequadas

Integração do sistema de estacionamento de bicicletas (paraciclos e bicicletários) com as calçadas, visando ao pleno acesso de ciclistas aos estabelecimentos

을 Alargamento e melhora das vias estruturais

Adequação de pontes, viadutos e passarelas para a travessia segura de pedestres e ciclistas

Implantação de travessias em nível em vias que não permitem interrupção de tráfego de veículos motorizados

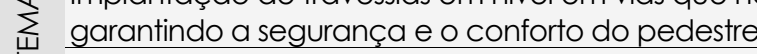

$\stackrel{5}{\sim}$ Implantação de estruturas de acalmamento de tráfego e redução de velocidade, especialmente em vias não estruturais, de forma a disciplinar o uso do espaço entre pedestres, bicicletas e veículos

Modernização da rede semafórica e aprimoramento da sinalização vertical e horizontal em todo o sistema viário

Instalação de mobiliário urbano nos passeios públicos e vias de pedestre

Recuperar, restaurar e fazer a manutenção de passeios públicos em áreas degradadas

Restrições e condicionantes à implantação de empreendimentos nos lotes lindeiros às vias estruturais, para garantir a fluidez do tráfego nas vias do sistema viário

Definição da declividade máxima das vias

Definição de largura mínima de canteiro central, passeio público, via de pedestre, ciclovia e leito carroçável Arborização e área permeável nas calçadas, obedecendo, para o plantio, o espaçamento mínimo e a especificação das espécies arbóreas

Nas áreas de influência dos eixos de transformação, em contrapartida à doação de área para alargamento de calçada, fica dispensado o recuo obrigatório de frente

Definição de taxa mínima de permeabilidade, taxa máxima de ocupação e coeficiente máximo de aproveitamento

Previsão de implementação de fachadas ativas no térreo dos edifícios

Articulação entre espaço público e espaço privado, por meio de estímulos à manutenção de espaços abertos

para fruição pública no pavimento de acesso às edificações, em especial nas centralidades existentes e nos

eixos de estruturação da transformação urbana

○ Contenção da verticalização

Diversificação das formas de implantação das edificações

iष Prever condições de conforto ambiental: insolação, aeração, permeabilidade do solo e índice mínimo de

U. cobertura vegetal

O Composição de conjuntos urbanos que superem exclusivamente o lote como unidade de referência de

configuração urbana, adotando também a quadra como referência de composição do sistema edificado

Aprimoramento do desenho urbano, ampliando e articulando os espaços de uso público, em especial os arborizados e destinados à circulação e ao bem-estar dos pedestres

Acessibilidade nas edificações e no espaço público

Instalação de galerias para uso compartilhado de serviços públicos

Limite de vedação do lote

Adequação da inserção de empreendimentos de médio e grande porte em relação ao entorno 
Tabela 3 - Continuação...

Propostas relacionadas ao desenho urbano - PDE (2014) e LPUOS (2016)

Combate à degradação ambiental: recuperar as fachadas em áreas degradadas, restaurá-las e fazer a manutenção delas

Identificação e preservação dos eixos histórico-culturais

$\stackrel{2}{\text { Preservação de eixos visuais que garantam sua apreensão pelos cidadãos }}$

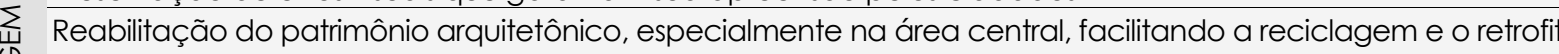

das edificações para novos usos

Combate à polvição visual: enterramento do cabeamento aéreo e ordenação da inserção de anúncios nos

espaços públicos, proibindo a publicidade

Para inserção de elementos na paisagem urbana, considerar as diversidade dos bairros, os bens culturais e

$\bigcirc$ ambientais de interesse de preservação, o sistema edificado e a infraestrutura

iष Qualificação ambiental da instalação de equipamentos sociais nas áreas verdes públicas: os fechamentos do

崩 lote por anteparo vertical não vedado, buscando permeabilidade visual

$\stackrel{\sim}{\alpha}$ Controle da volumetria das edificações no lote e na quadra a fim de evitar interferências negativas na paisagem urbana

Estímulo à promoção de edifícios de uso misto e à utilização do térreo dos edifícios para usos não residenciais, por meio de incentivos de outorga onerosa ou outros benefícios, em especial nos eixos de transformação urbana

Parâmetros de incomodidade: estabelecer limites quanto à interferência de atividades não residenciais em

@ relação ao uso residencial

Compatibilização dos usos do solo com as condicionantes de relevo, geológico-geotécnicas, com a

legislação de proteção aos mananciais e recuperação deles e com a preservação de bens e áreas de valor

ऽ histórico, paisagístico, arqueológico, cultural e religioso

Limites mínimos e máximos de área construída computável destinada a estacionamento de veículos,

condicionando o número máximo à compensação urbanística por sua utilização

○ Definição do parcelamento do solo com dimensões mínimas e máximas de lotes e quadras

Compatibilização das tipologias de parcelamento do solo com as condicionantes de relevo, geológico-

을 geotécnicas, com a legislação de proteção aos mananciais e recuperação deles e com a preservação de

Щ

\& O Doação mínima de área verde e área para equipamento proporcional a área total da gleba ou lote, nos

यु casos em que o parcelamento não for exigido pela LPUOS

Remembramento de lotes, englobando dimensões máximas do lote resultante e previsão das condições para

a destinação de áreas públicas

Ampliação de parques urbanos e lineares para equilibrar a relação entre o ambiente construído e as áreas verdes e livres

Articulação das áreas verdes significativas, espaços livres e parques urbanos e lineares, por meio de caminhos

de pedestres e ciclovias preferencialmente nos fundos de vale

岁 Requalificação dos sistemas ambientais da cidade, considerando as infraestruturas de saneamento e

W drenagem, a partir da constituição e da articulação de espaços livres

$\sum$ Revisão dos perímetros dos parques propostos para integração das cabeceiras de drenagem e áreas com

vegetação significativa, visando à conectividade entre parques e áreas verdes e ao estabelecimento de

$\stackrel{\bigcirc}{\Psi}$ corredores ecológicos

¿ consumo de água e de energia, otimizar a utilização do espaço público e contribuir para a melhoria das condições ambientais

Quota ambiental e taxa de permeabilidade mínima: promover a qualificação ambiental, em especial a melhoria da retenção e infiltração da água nos lotes, a melhoria do microclima e a ampliação da vegetação LEGENDA:

Presente no Plano Diretor e na Lei de Parcelamento, Uso e Ocupação do Solo

Presente no Plano Diretor

Presente na Lei de Parcelamento, Uso e Ocupação do Solo

Fonte: elaboração dos autores com base no PDE e na LPUOS (2018).

Entre as estratégias relacionadas ao desenho urbano, identificadas na Tabela 3, a maioria se refere à infraestrutura de transporte e à ocupação do solo. As propostas voltadas à ocupação do solo, que aparecem tanto no PDE como na LPUOS, também possuem conexão com o sistema viário, uma vez que são diretrizes para eixos de transporte público, como o incentivo à fachada ativa e o espaço de fruição pública. Isso reforça a presença de estratégias relacionadas ao sistema viário e à qualificação de espaços para o pedestre. Nas propostas para o sistema viário, estão o alargamento e a qualificação de calçadas, a 
integração entre meios de transporte motorizados e não motorizados, a implantação de travessias para pedestres e bicicletas e as medidas de acalmamento de tráfego.

As propostas de ocupação do solo trazem os tradicionais parâmetros de regulação do solo urbano (recuos, taxa de permeabilidade e coeficiente de aproveitamento), no entanto são complementadas por estratégias que inovam em relação aos parâmetros tradicionais. 0 incentivo à diversificação na forma de implantação dos edifícios na quadra, a contenção da verticalização e a quadra como unidade referencial para projetos buscam a qualificação do perímetro das quadras e dos espaços entre edificações. A interface entre o espaço público e o privado também é o campo de atuação da fachada ativa e dos espaços de fruição pública. Essas propostas refletem na qualificação dos espaços públicos configurados pelo sistema viário.

A proteção da paisagem urbana também está associada a essa qualificação, a exemplo da proposta de enterramento de cabos em vias específicas. No entanto, conforme já apontado, questões estéticas podem ter menos influência na caminhabilidade do que outras medidas relacionadas com a estruturação do sistema viário (Boarnet et al., 2011). O controle de vagas destinadas a veículos, com a definição de quantidade máxima de vagas, reforça um processo de reversão da escala do automóvel e converge para uma das principais temáticas abordadas pelos artigos (Figura 5). As questões ambientais também estão presentes nas propostas de ambas as leis: o aumento das áreas verdes e permeáveis e o tratamento adequado das áreas de fundo de vale e cabeceiras de drenagem possuem o objetivo de melhorar o microclima urbano e a gestão das águas pluviais, bem como a formação de corredores ecológicos, que está em sintonia com as temáticas 3, 4, 5 e 7 em destaque nos artigos (Figura 5).

\section{Discussão e considerações finais}

Diante do que foi apresentado, é possível observar que algumas obras, desde a década de 1960, tiveram influência sobre o desenvolvimento do campo disciplinar do desenho urbano no Brasil em resposta aos novos desafios nas cidades contemporâneas. Está posta uma tendência de crescente valorização da escala do pedestre no processo de planejamento urbano, que culmina em estratégias cada vez mais difundidas relacionadas ao desenho do espaço urbano, com o objetivo de promover a vida urbana. As publicações internacionais traduzidas para o português e editadas nacionalmente tiveram um impacto nessa configuração. Desde as obras clássicas, como as de Kevin Lynch (1960), Gordon Cullen (1983) e Jane Jacobs (2000), até aquelas mais recentes e difundidas nos meios profissionais, como as de Jan Gehl $(2015,2018)$ e Douglas Farr $(2013)$, todas tiveram um papel relevante para a formação e a construção de um pensamento sobre o desenho urbano no Brasil, ainda que sob pesquisas e experiências internacionais, estabelecidas em outra realidade social. De qualquer forma, é importante dizer que, em compasso com uma produção nacional que se lançou a partir dos vários seminários de desenho urbano ocorridos no Brasil (Turkienicz, 1984, 1986) até a sistematização feita por Vicente Del Rio (1990), que, mais recentemente, focalizou o que poderia ser chamado de traços de desenho urbano contemporâneo no Brasil (Del Rio \& Siembieda, 2013), essas obras configuraram uma matriz de pensamento que, de alguma forma, está presente nas reflexões sobre a qualidade de vida na cidade, o que reflete na série de manuais acerca de passeios públicos que passaram a ser publicados por prefeituras em diversos municípios do Brasil.

Em relação ao método aplicado a esta pesquisa, foram constatados alguns pontos que devem ser ressaltados. Primeiramente, vale observar que o campo do desenho urbano se associou, em certa medida, com assuntos relacionados à esfera ambiental, ecológica e sustentável, considerando a quantidade de artigos que trazem esses termos no seu conjunto de palavras-chave. É algo que não chega a surpreender, mas que revela uma compreensão de que as ferramentas disciplinares do desenho urbano podem representar algumas chaves para a melhoria da qualidade ambiental nas cidades. 0 crescimento das publicações científicas relacionadas ao tema é um aspecto que deve ser destacado (Figura 2). Apesar de irregular, é notável um aumento das publicações a partir, principalmente, de 
2007, o que revela um campo investigativo em ascensão, recente e certamente estabelecido a partir do revigoramento das problemáticas relacionadas à qualidade espacial das cidades, sua urbanidade e suas condições para tornar o espaço mais adequado às atividades humanas de forma mais sustentável.

A associação entre o tema do desenho urbano ao uso e à ocupação do solo demonstrou com clareza que a questão da mobilidade urbana tem absoluta vinculação nessa associação. Supõe-se, nesse sentido, que o ambiente construído e seus usos nos lotes pressupõem uma organização do sistema de mobilidade e que o desenho urbano pode recuperar essa combinação. A relação entre o meio construído e as opções de meios de deslocamento se destacou também nesse conjunto de publicações, demonstrando uma nítida preocupação em compreender as razões pelas quais essas escolhas são feitas. Nesse aspecto, reforça-se o entendimento de que o desenho urbano trabalha na interface entre os espaços público e privado, entre o espaço construído (fixo) e o espaço de circulação (fluxos), na intermediação de fatores comportamentais.

A revisão do PDE e da LPUOS em São Paulo, mesmo que referenciada em novos conceitos, seguiu, em certa medida, uma estrutura ancorada nos documentos anteriores. Permanecem ainda os tradicionais mecanismos e parâmetros urbanísticos, como coeficiente de aproveitamento e recuos. As propostas "inovadoras" ali incluídas, por vezes, não são facilmente aplicáveis e realizáveis, mas demonstram a introdução de preocupações antes não presentes. É visível que, a partir de um meio ou de outro (ou pelos meios científicos ou pela via da disseminação ampla desse conhecimento), tais questões estão sendo colocadas em pauta e vêm apresentando certa aderência aos mecanismos de intervenção. No caso do PDE e da LPUOS de São Paulo, foi possível encontrar várias dessas acepções, podendo revelar essa sinergia.

As propostas dos planos relacionadas à infraestrutura de transporte, tanto pela quantidade como por seu conteúdo, corroboram essa afirmação e, de fato, evidenciam que esses instrumentos estão vinculados a um novo momento de articulação com o conhecimento no campo do urbanismo. Nas propostas referentes ao uso e à ocupação do solo, por exemplo, a composição de conjuntos urbanos que superem exclusivamente o lote como unidade de referência de configuração urbana, o estabelecimento de limites mínimos e máximos de área construída computável destinada a estacionamento de veículos, ou mesmo a possibilidade de o passeio ganhar mais largura em compensação à dispensa de recuo frontal das edificações no lote têm clara sintonia com alguns dos artigos selecionados na terceira etapa da pesquisa. Uma das diretrizes que se tornou mais conhecida, a fachada ativa, está justamente associada a uma melhor relação entre a edificação, o seu uso lindeiro e a circulação de pedestres, mecanismo esse que se coloca em meio a um certo debate em torno da tese Jane Jacobs, na defesa do uso misto e da densidade como promotor de caminhabilidade, conceito defendido também por artigos descobertos pela pesquisa (Boulange et al., 2017; Lee et al., 2017; Gunn et al., 2017; Lee, 2015; Lerman \& Omer, 2016; Koohsari, et al., 2016; Choi, 2014; Johnson \& Marko, 2008; Krizek, 2003; Greenwald, 2003; Handy et al., 2002), ou, ao contrário, a crítica de que o uso misto e as maiores densidades populacionais geram a caminhabilidade, ao serem apontadas limitações nessa relação (Maat et al., 2005; Kemperman \& Timmermans, 2009; Lee, 2015).

As aproximações entre a produção científica no campo do desenho urbano identificadas aqui por meio do método da RSL e a concepção da nova legislação urbanística de São Paulo desenvolvidas neste artigo vêm de encontro a muitos dilemas que se colocam nessa relação entre ciência e sua aplicação. Em que medida as descobertas e os achados têm sido apropriados e aplicados às novas realidades das cidades contemporâneas no Brasil? Para além do velho discurso da boa qualidade urbana, há que se debruçar mais sobre as variáveis e os aspectos intrínsecos a essa qualidade, identificando-se os atributos necessários para que ela seja atingida. Para além também de mecanismos tradicionais e nem sempre muito eficazes, como a legislação urbanística, esta discussão deve transcender os meios tradicionais de aplicação dos conhecimentos no Brasil, que, no âmbito do planejamento, pautaram-se com muita veemência por meio da legislação, na distopia de uma sociedade que se nega, muitas vezes, a aceitar, de fato, a construção dessa qualidade. 


\section{Referências}

Abdel-Salam, H. (1996). Perception of qualitative changes in public open spaces: the case of Alexandria, Egypt. URBAN DESIGN International, 1(1), 27-40. http://dx.doi.org/10.1057/udi.1996.4.

Abe, D. (2003). Features of initial framework for urban regeneration policies at the historic center of Barcelona. In Proceedings of the Annual Conference of the City Planning Institute of Japan (Vol. 99). Tokyo: The City Planning Institute of Japan. https://doi.org/10.11361/cpij1.38.0.99.0.

Aelbrecht, P. S. (2010). Rethinking urban design for a changing public life. Journal of Place Management and Development, 3(2), 113-129. http://dx.doi.org/10.1108/17538331011062667.

Aguilera-Martínez, F. A., Medina-Ruiz, M., Castellanos-Escobar, M. C., \& Perilla-Agudelo, K. J. (2017). Intervención social en el borde urbano desde el proceso de la significación cultural. Revista de Arquitectura, 19(2), 78-93. http://dx.doi.org/10.14718/RevArq.2017.19.2.1495.

Al-Bishawi, M., Ghadban, S., \& Jørgensen, K. (2015). Women's behaviour in public spaces and the influence of privacy as a cultural value: the case of Nablus, Palestine. Urban Studies, 54(7), 1559-1577. https://doi.org/10.1177/0042098015620519.

Allweil, Y., \& Kallus, R. (2013). Re-forming the political body in the city: the interplay of male bodies and territory in urban public spaces in Tel Aviv. City, 17(6), 748-777. http://dx.doi.org/10.1080/13604813.2013.849128.

Al-Saffar, M. (2016). Toward an integrated sustainable urban design framework in the historic center of Baghdad. The International Journal of Environmental Sustainability, 13, 31-52.

Alves, F., Abrantes, N., \& Abrantes, V. (2009). Getting urban profits through the freedom of use. International Journal for Housing Science and Its Applications, 33, 127-135.

Anderson, J., Ruggeri, K., Steemers, K., \& Huppert, F. (2016). Lively social space, well-being activity, and urban design: findings from a low-cost community-led public space intervention. Environment and Behavior, 49(6), 685716. http://dx.doi.org/10.1177/0013916516659108.

Appleyard, B. (2012). Sustainable and healthy travel choices and the built environment: analyses of green and active access to rail transit stations along individual corridors. Transportation Research Record: Journal of the Transportation Research Board, 2303(1), 38-45. http://dx.doi.org/10.3141/2303-05.

Araújo, M. R. M., Oliveira, J. M., Jesus, M. S., Sá, N. R., Santos, P. A. C., \& Lima, T. C. (2011). Transporte público coletivo: discutindo acessibilidade, mobilidade e qualidade de vida. Psicologia e Sociedade, 23(3), 574-582. http://dx.doi.org/10.1590/S0102-71822011000300015.

Ashley, R., Lundy, L., Ward, S., Shaffer, P., Walker, L., Morgan, C., Saul, A., Wong, T., \& Moore, S. (2013). Watersensitive urban design: opportunities for the UK. Proceedings of the Institution of Civil Engineers. Municipal Engineer, 166(2), 65-76. http://dx.doi.org/10.1680/muen.12.00046.

Asl, A., Haghlesan, M., \& Taraf, A. (2012). The role of pedestrian streets in sustainability of urban spaces, case study: Tabriz Tarbiyat Street, Iran 1. Advances in Natural and Applied Sciences, 6, 1014-1021.

Atkinson, C. (2015). A thick green line: extracting urban space from vehicle infrastructure. Spaces \& Flows: an International Journal of Urban \& Extra Urban, 6(3), 1-14.

Aydin, M. Ç., \& Ayataç, H. (2015). A pedesterianization case in the context of public interest: a pedesterianization project in Eminönü historical peninsula (hobyar neighborhood and surroundings). A/Z ITU Journal of Faculty of Architecture, 12(1), 3-13.

Badiozamani, G. (2003). Car-free days: a shift in the planning paradigm? Natural Resources Forum, 27(4), 300-303. http://dx.doi.org/10.1111/j.0165-0203.2003.00064.x.

Badland, H., \& Schofield, G. (2005). Transport, urban design, and physical activity: an evidence-based update. Transportation Research Part D, Transport and Environment, 10(3), 177-196.

http://dx.doi.org/10.1016/j.trd.2004.12.001. 
Badland, H., Schofield, G., \& Garrett, N. (2008). Travel behavior and objectively measured urban design variables: associations for adults traveling to work. Health \& Place, 14(1), 85-95.

http://dx.doi.org/10.1016/j.healthplace.2007.05.002. PMid:17590378.

Bajšanski, I., Milošević, D., \& Savic, S. (2015). Evaluation and improvement of outdoor thermal comfort in urban areas on extreme temperature days: applications of automatic algorithms. Building and Environment, 94, 94. http://dx.doi.org/10.1016/j.buildenv.2015.10.019.

Balsas, C. J. L. (2017). The right to walk in cities, a comparative review of Macau, Lisbon and Las Vegas. International Journal of Law in the Built Environment, 9(6). http://dx.doi.org/10.1108/IJLBE-03-2017-0012.

Balsas, J. L. (2007). City centre revitalization in Portugal: a study of Lisbon and Porto. Journal of Urban Design, 12(2), 231-259. http://dx.doi.org/10.1080/13574800701306328.

Barros, M. P., Nogueira, M. C. J. A., \& Musis, C. R. (2010). O projeto de parque urbano e os riscos da exposição ao calor. Ambiente Construído, 10(2), 147-156. https://dx.doi.org/10.1590/S1678-86212010000200010.

Bejleri, I., Roaza, R., Thomas, A., Turton, T., \& Zwick, P. (2003). Florida's efficient transportation decision-making process: Laying the Technology Foundation. Transportation Research Record: Journal of the Transportation Research Board, 1859(1), 19-28. http://dx.doi.org/10.3141/1859-03.

Bekessy, S. A., White, M., Gordon, A., Moilanen, A., Mccarthy, M. A., \& Wintle, B. A. (2012). Transparent planning for biodiversity and development in the urban fringe. Landscape and Urban Planning, 108(2-4), 140-149.

http://dx.doi.org/10.1016/j.landurbplan.2012.09.001.

Biddulph, M. (1999). Bringing vitality to a campus environment. URBAN DESIGN International, 4(3-4), 153-166. http://dx.doi.org/10.1057/udi.1999.22.

Boarnet, M. G., Forsyth, A., Day, K., \& Oakes, J. M. (2011). The street level built environment and physical activity and walking: results of a predictive validity study for the irvine minnesota inventory. Environment and Behavior, 43(6), 735-775. http://dx.doi.org/10.1177/0013916510379760.

Boarnet, M., \& Crane, R. (2001). The influence of land use on travel behavior: specification and estimation strategies. Transportation Research Part A, Policy and Practice, 35(9), 823-845. http://dx.doi.org/10.1016/S09658564(00)00019-7.

Borst, A. (2008). Regenerative French urbanism: sustainable planning strategies in Lille, Lyon, and Montpellier. Journal of Green Building, 3(3), 79-87. http://dx.doi.org/10.3992/jgb.3.3.79.

Boulange, C., Gunn, L., Giles-Corti, B., Mavoa, S., Pettit, C., \& Badland, H. (2017). Examining associations between urban design attributes and transport mode choice for walking, cycling, public transport and private motor vehicle trips. Journal of Transport \& Health, 6, 155-166. http://dx.doi.org/10.1016/j.jth.2017.07.007.

Brasil. (2001, 10 de julho). Lei $n^{\circ}$ 10.257, de 10 de julho de 2001. Regulamenta os arts. 182 e 183 da Constituição Federal, estabelece diretrizes gerais da política urbana e dá outras providências. Brasília: Diário Oficial da União.

Brereton, P., Kitchenham, B. A., Budgen, D., Turner, M., \& Khalil, M. (2007). Lessons from applying the systematic literature review process within the software engineering domain. Journal of Systems and Software, 80(4), 571583. http://dx.doi.org/10.1016/j.jss.2006.07.009.

Budds, J., \& Teixeira, P. (2005). Ensuring the right to the city: pro-poor housing, urban development and tenure legalization in São Paulo, Brazil. Environment and Urbanization, 17(1), 89-114. http://dx.doi.org/10.1630/0956247053633809.

Buxton, M., \& Scheurer, J. (2007). Density and outer urban development in Melbourne. Urban Policy and Research, 25(1), 91-111. http://dx.doi.org/10.1080/08111140701222831.

Cárdenas O’Byrne, S. (2017). Medir el uso del espacio público urbano seguro. Society and Economy, 33, 1-33. http://dx.doi.org/10.25100/sye.v0i33.5620. 
Cárdenas O’Byrne, S., \& Verónica D’Inca, M. (2016). Arquitectura de la noviolencia: el papel del otro en la construcción del espacio común. Polis (Santiago), 15(43), 251-269. http://dx.doi.org/10.4067/S071865682016000100012.

Carmona, M., Heath, T., Oc, T., \& Tiesdell, S. (2003). Public places - urban spaces: the dimension of urban design. Oxford: Architectural Press.

Cervero, R. (2002). Built environments and mode choice: toward a normative framework. Transportation Research Part D, Transport and Environment, 7(4), 265-284. http://dx.doi.org/10.1016/S1361-9209(01)00024-4.

Cervero, R. (2007). Transit-oriented development's ridership bonus: a product of self-selection and public policies. Environment \& Planning A, 39(9), 2068-2085. http://dx.doi.org/10.1068/a38377.

Cervero, R. (2009). Transport infrastructure and global competitiveness: balancing mobility and livability. The Annals of the American Academy of Political and Social Science, 626(1), 210-225. http://dx.doi.org/10.1177/0002716209344171.

Chamberlain, L. (2017). Zoning at 100. American Planning Association, 83, 44-49.

Chan, E., \& Lee, K. L. (2009). Design considerations for environmental sustainability in high density development: a case study of Hong Kong. Environment, Development and Sustainability, 11(2), 359-374. http://dx.doi.org/10.1007/s10668-007-9117-0.

Chatterjee, R. (2009). Smart growth: a solution to climate change? Environmental Science \& Technology, 43(6), 1660. http://dx.doi.org/10.1021/es900293g. PMid:19368152.

Choi, E. (2014). Walkability and the complexity of walking behavior. A/Z ITU Journal of the Faculty of Architecture, 11(2), 87-99. Recuperado em 5 de agosto de 2018, de http://urn.kb.se/resolve?urn=urn:nbn:se:kth:diva-161539

Chong, H., Yow, W. Q., Loo, D., \& Patrycia, F. (2015). Psychosocial well-being of the elderly and their perception of matured estate in Singapore. Journal of Housing for the Elderly, 29(3), 259-297.

http://dx.doi.org/10.1080/02763893.2015.1055025.

Chow, A. (2014). Urban design, transport sustainability and residents' perceived sustainability: a case study of transit-oriented development in Hong Kong. Journal of Comparative Asian Development, 13(1), 73-104.

http://dx.doi.org/10.1080/15339114.2014.892818.

Coordenação de Aperfeiçoamento de Pessoal de Nível Superior - CAPES. (2018). Portal Periódicos CAPES.

Recuperado em 20 de dezembro de 2018, de http://www.periodicos.capes.gov.br

Crane, R. (2010). The Influence of Urban Form on Travel: An Interpretive Review. Journal of Planning Literature, 15(1), 3-23. http://dx.doi.org/10.1177/08854120022092890.

Craun, Z. (2012). Refurbishment as a sustainable Urban Design strategy. Journal of International Affairs, 65(2), 157-167.

Cullen, G. (1983). Paisagem urbana. São Paulo: Martins Fontes.

Dagenhart, R., Leigh, N., \& Skach, J. (2006). Brownfields and urban design: learning from Atlantic Station. WIT Transactions on Ecology and the Environment, 94, 185-194. http://dx.doi.org/10.2495/BF060181.

Davis, D. (2013). Zero-tolerance policing, stealth real estate development, and the transformation of public space evidence from Mexico city. Latin American Perspectives, 40(2), 53-76.

http://dx.doi.org/10.1177/0094582X12467761.

Del Castillo Oyarzún, M., \& Castillo Haeger, C. (2014). Aproximación bioclimática para el diseño de espacios públicos, análisis inicial en distintas plazas chilenas. Arquitectura y Urbanismo, 35(3), 69-82.

Del Rio, V. (1990). Introdução ao desenho urbano no processo de planejamento. São Paulo: Pini.

Del Rio, V., \& Siembieda, W. (2013). Desenho urbano contemporâneo no Brasil. Rio de Janeiro: Grupo Gen. 
Djukic, A., \& Vukmirovic, M. (2012). Redesigning the network of pedestrian Spaces in the function of reduction of CO2 Emission. Case study: pančevo and Vršac. Spatium (Belgrade), 27(27), 31-39.

http://dx.doi.org/10.2298/SPAT1227031D.

Djukic, A., Vukmirovic, M., \& Stankovic, S. (2016). Principles of climate sensitive urban design analysis in identification of suitable urban design proposals. Case study: central zone of Leskovac competition. Energy and Building, 115, 23-35. http://dx.doi.org/10.1016/j.enbuild.2015.03.057.

Doeksen, H. (1997). Reducing crime and the fear of crime by reclaiming New Zealand's suburban street. Landscape and Urban Planning, 39(2-3), 243-252. http://dx.doi.org/10.1016/S0169-2046(97)00057-1.

Dovey, K., \& Pafka, E. (2017). What is functional mix? An assemblage approach. Planning Theory \& Practice, 18(2), 249-267. http://dx.doi.org/10.1080/14649357.2017.1281996.

Dovey, K., \& Symons, F. (2014). Density without intensity and what to do about it: reassembling public/private interfaces in Melbourne's Southbank hinterland. Australian Plants, 51(1), 34-46.

http://dx.doi.org/10.1080/07293682.2013.776975.

Dukanovic, Z., \& Zivkovic, J. (2015). Public art \& public space programme: learning, but doing!. Annales. Series Historia et Sociologia, 25(1), 49-64.

Dymitrow, M. (2014). The effigy of urbanity or a rural parody? A visual approach to small-town public space. Journal of Cultural Geography, 31(1), 1-31. http://dx.doi.org/10.1080/08873631.2013.873298.

Eliasson, I., Knez, I., Westerberg, U., Thorsson, S., \& Lindberg, F. (2007). Climate and behaviour in a Nordic city. Landscape and Urban Planning, 82(1-2), 72-84. http://dx.doi.org/10.1016/j.landurbplan.2007.01.020.

Elinbaum, P. (2014). Plan y proyecto territorial en las Comarcas Centrales de Cataluña: herramientas de ordenación para una nueva escala de ciudad. EURE (Santiago), 40(121), 225-245.

http://dx.doi.org/10.4067/S0250-71612014000300011.

Elsheshtawy, Y. (2015). Observing the public realm: William Whyte's the social life of small urban spaces. Built Environment, 41(3), 399-411. http://dx.doi.org/10.2148/benv.41.3.399.

Ercoskun, Y., \& Karaaslan, S. (2011). Next-generation urban design guides for sustainability of small towns: a case study on Gudul, Turkey. URBAN DESIGN International, 16(2), 16. http://dx.doi.org/10.1057/udi.2011.3.

Ewing, R. (2016). Contribution of urban design qualities to pedestrian activity. American Planning Association, 82(2), 50-51.

Ewing, R., \& Cervero, R. (2001). Travel and the built environment: a synthesis. Transportation Research Record: Journal of the Transportation Research Board, 1780(1), 87-114. http://dx.doi.org/10.3141/1780-10.

Farr, D. (2013). Urbanismo sustentável. Desenho urbano com a natureza. Porto Alegre: Bookman.

Ferguson, K. (2012). A unified model for integral city design. Management of Environmental Quality, 23(2), 140149. http://dx.doi.org/10.1108/14777831211204895.

Fernandez Mucoz, L. (2015). The experience of urban walking as a guidance for urban quality assessment. SGEM2015 Conference Proceedings, 4(1), 693-700. http://dx.doi.org/10.5593/SGEMSOCIAL2015/B41/S15.085.

Ford, R. (2008). World cities and global change: observations on monumentality in urban design. Eurasian Geography and Economics, 49(3), 237-262. http://dx.doi.org/10.2747/1539-7216.49.3.237.

Frick, D. (2007). Spatial synergy and supportiveness of public space. Journal of Urban Design, 12(2), 261-274. http://dx.doi.org/10.1080/13574800701306369.

Fryd, O., Backhaus, A., Birch, H., Fratini, C., Ingvertsen, S., Jeppesen, J., Panduro, T. E., Roldin, M., \& Jensen, M. (2013). Water sensitive urban design retrofits in Copenhagen - 40\% to the sewer, $60 \%$ to the city. Water Science and Technology: a Journal of the International Association on Water Pollution Research, 67(9), 1945-1952.

http://dx.doi.org/10.2166/wst.2013.073. 
Furuseth, O. J. (1997). Neotraditional planning: a new strategy for building neighborhoods? Land Use Policy, 14(3), 201-2013. http://dx.doi.org/10.1016/S0264-8377(97)00002-1.

Gaffikin, F., Mceldowney, M., \& Sterrett, K. (2010). Creating shared public space in the contested city: the role of urban design. Journal of Urban Design, 15(4), 493-513. http://dx.doi.org/10.1080/13574809.2010.502338.

Gago, J., Roldan, J., Pacheco-Torres, R., \& Ordóñez, J. (2013). The city and urban heat islands: A review of strategies to mitigate adverse effects. Renewable \& Sustainable Energy Reviews, 25, 749-758.

http://dx.doi.org/10.1016/j.rser.2013.05.057.

Gehl, J. (2015). Cidades para pessoas. (3. ed.). São Paulo: Perspectiva.

Gehl, J. (2018). A vida na cidade: como estudar. São Paulo: Perspectiva.

Giddings, B., Charlton, J., \& Horne, M. (2011). Public squares in European city centres. Urban Design International, 16(3), 202-212. http://dx.doi.org/10.1057/udi.2011.6.

Giraldo, G. H. (2015). Producción social, proceso participativo e intervención sostenible en el espacio público de los centros históricos. El caso de Pamplona, Colombia. Territorios, 33, 33-61.

http://dx.doi.org/10.12804/territ33.2015.02.

Goins, K. V., Ye, J., Leep, C. J., Robin, N., \& Lemon, S. C. (2016). Local health department engagement in community physical activity policy. American Journal of Preventive Medicine, 50(1), 57-68.

http://dx.doi.org/10.1016/j.amepre.2015.06.033. PMid:26410187.

Gonçalves, A., \& Meneguetti, K. S. (2015). Projeto de arborização como patrimônio da cidade. Ambiente Construído, 15(1), 99-118. http://dx.doi.org/10.1590/S1678-86212015000100009.

Greenwald, M. J. (2003). The road less traveled: new urbanist inducements to travel mode substitution for nonwork trips. Journal of Planning Education and Research, 23(1), 39-57.

http://dx.doi.org/10.1177/0739456X03256248.

Grgic, A., Barcot, M., \& Tusek, D. (2014). Street as a paradigm of pleasant life - influence of the mediaeval city within diocletian's palace on the new urban typology of modern Split. SGEM2014 Conference Proceedings, 4(1), 1097-1104. http://dx.doi.org/10.5593/SGEMSOCIAL2014/B41/S15.133.

Gunn, L. D., Mavoa, S., Boulangé, C., Hooper, P., Kavanagh, A., \& Giles-Corti, B. (2017). Designing healthy communities: creating evidence on metrics for built environment features associated with walkable neighbourhood activity centres. The International Journal of Behavioral Nutrition and Physical Activity, 14(1), 164. http://dx.doi.org/10.1186/s12966-017-0621-9. PMid:29202849.

Guttenplan, M., Davis, B., Steiner, R., \& Miller, D. (2003). Planning-level areawide multimodal level-of-service analysis: performance measures for congestion management. Transportation Research Record: Journal of the Transportation Research Board, 1858(1), 61-68. http://dx.doi.org/10.3141/1858-09.

Ha, E., Joo, Y., \& Jun, C. (2011). An empirical study on sustainable walkability indices for transit-oriented development by using the analytic network process approach. International Journal of Urban Sciences, 15(2), 137146. http://dx.doi.org/10.1080/12265934.2011.615977.

Haas, T. (2009). Traditional European squares in contemporary urbanism: dubrovnik's medieval squares. Open House International, 34(4), 57-73.

Habib, F., Peimani, N., \& Daroudi, M. R. (2013). Urban deteriorated fabric regeneration according to public open space enhancement (case study: tabriz). World Applied Sciences Journal, 21(8), 1237-1249.

http://dx.doi.org/10.5829/idosi.wasj.2013.21.8.119.

Hall, A. C. (2000). A new paradigm for local development plans. Urban Design Internacional, 5(2), 123-140. https://doi.org/10.1057/palgrave.udi.9000019.

Hall, A. C. (2008). The form-based development plan: bridging the gap between theory and practice in urban morphology. Urban Morphology, 12(2), 77-95. Recuperado em 20 de julho de 2018, de 
https://www.scopus.com/inward/record.uri?eid=2-s2.0-

54749115245\&partnerID=40\&md5=a3c081bbbf43c0cd505e6e22d1fd41a8

Hamada, S., Tanaka, T., \& Ohta, T. (2013). Impacts of land use and topography on the cooling effect of green areas on surrounding urban areas. Urban Forestry \& Urban Greening, 12(4), 426-434.

http://dx.doi.org/10.1016/j.ufug.2013.06.008.

Hamilton-Baillie, B. (2004). Urban design: why don't we do it in the road? Modifying traffic behavior through legible urban design. Journal of Urban Technology, 11(1), 43-62.

http://dx.doi.org/10.1080/1063073042000341970.

Handy, S. L., Boarnet, M. G., Ewing, R., \& Killingsworth, R. E. (2002). How the built environment affects physical activity: views from urban planning. American Journal of Preventive Medicine, 23(2, Suppl), 64-73. http://dx.doi.org/10.1016/S0749-3797(02)00475-0. PMid:12133739.

Heffernan, E., Heffernan, T., \& Pan, W. (2014). The relationship between the quality of active frontages and public perceptions of public spaces. URBAN DESIGN International, 19(1), 92-102. http://dx.doi.org/10.1057/udi.2013.16.

Huang, A., Gallegos, L., \& Lerman, K. (2017). Travel analytics: understanding how destination choice and business clusters are connected based on social media data. Transportation Research Part C, Emerging Technologies, 77, 245-256. http://dx.doi.org/10.1016/j.trc.2016.12.019.

Inam, A. (2002). Meaningful urban design: teleological/catalytic/relevant. Journal of Urban Design, 7(1), 35-58. http://dx.doi.org/10.1080/13574800220129222.

Jackson, L. (2003). The relationship of urban design to human health and condition. Landscape and Urban Planning, 64(4), 191-200. http://dx.doi.org/10.1016/S0169-2046(02)00230-X.

Jacobs, J. (2000). Morte e vida de grandes cidades. São Paulo: Martins Fontes.

Jamei, E., Rajagopalan, P., Seyedmahmoudian, M., \& Jamei, Y. (2015). Review on the impact of urban geometry and pedestrian level greening on outdoor thermal comfort. Renewable \& Sustainable Energy Reviews, 54, 1002-1017. https://doi.org/10.1016/j.rser.2015.10.104.

Johnson, S., \& Marko, J. (2008). Designing healthy places: land use planning and public health. Environments, 35(3), 9-19. Recuperado em 20 de dezembro de 2018, de https://www.scopus.com/inward/record.uri?eid=2-s2.068249103617\&partnerID=40\&md5=3a4535515eca0460e9169ceb0816b6b5

Jou, K. K. (2011). Evaluating integration between public transportation and pedestrian-oriented urban spaces in two main metro stations of Tehran. Scientific Research and Essays, 6(13), 2695-2709.

Kamete, A. Y. (1999). Restrictive control of urban high-density housing in Zimbabwe: Deregulation, challenges and implications for urban design, housing. Theory and Society, 16(3), 136-151.

Kemperman, A., \& Timmermans, H. (2009). Influences of built environment on walking and cycling by latent segments of aging population. Transportation Research Record: Journal of the Transportation Research Board, 2134(1), 1-9. http://dx.doi.org/10.3141/2134-01.

Kenworthy, J. (2007). Urban planning and transport paradigm shifts for cities of the post-petroleum age. Journal of Urban Technology, 14(2), 47-70. http://dx.doi.org/10.1080/10630730701531708.

Kim, T., Sohn, D.-W., \& Choo, S. (2016). An analysis of the relationship between pedestrian traffic volumes and built environment around metro stations in Seoul. KSCE Journal of Civil Engineering, 21(4), 1443-1452. http://dx.doi.org/10.1007/s12205-016-0915-5.

Kitchenham, B., Pearl Brereton, O., Budgen, D., Turner, M., Bailey, J., \& Linkman, S. (2009). Systematic literature reviews in software engineering: a systematic literature review. Information and Software Technology, 51(1), 7-15. http://dx.doi.org/10.1016/j.infsof.2008.09.009.

Kleerekoper, L., van Esch, M., \& Salcedo, T. B. (2011). How to make a city climate-proof, addressing the urban heat island effect. Resources, Conservation and Recycling, 64, 30-38. https://doi.org/10.1016/j.resconrec.2011.06.004. 
Koohsari, M. J., Badland, H., \& Giles-corti, B. (2013). (Re)Designing the built environment to support physical activity: bringing public health back into urban design and planning. Cities (London, England), 35, 294-298. http://dx.doi.org/10.1016/j.cities.2013.07.001.

Koohsari, M. J., Owen, N., Cerin, E., Giles-Corti, B., \& Sugiyama, T. (2016). Walkability and walking for transport: characterizing the built environment using space syntax. The International Journal of Behavioral Nutrition and Physical Activity, 13(1), 1-9. http://dx.doi.org/10.1186/s12966-016-0448-9. PMid:27881173.

Kopic, M., \& Kurtović-Folić, N. (2011). Principles of the design of urban space that support rail systems from the aspect of urban elements and elements of the relief. Geodetski Vestnik, 55, 726-749.

Krizek, K. J. (2003). Operationalizing neighborhood accessibility for land use - Travel behavior research and regional modeling. Journal of Planning Education and Research, 22(3), 270-287. http://dx.doi.org/10.1177/0739456X02250315.

Krüger, P. E., Drach, P. B., \& Bröde, P. (2015). Implications of air-conditioning use on thermal perception in open spaces: a field study in downtown Rio de Janeiro. Building and Environment, 94, 417-425.

http://dx.doi.org/10.1016/j.buildenv.2015.07.024.

Lajut, J. (2016). Análise do impacto do novo plano diretor estratégico da cidade de São Paulo no planejamento de produtos residenciais (Dissertação de mestrado). Faculdade de Arquitetura e Urbanismo, Universidade de São Paulo, São Paulo.

Lamas, J. M. R. G. (2004). Morfologia urbana e desenho da cidade (3. ed.). Lisboa: Fundação Calouste Gulbenkian.

Lee, J. (2013). Perceived neighborhood environment and transit use in low-income populations. Transportation Research Record: Journal of the Transportation Research Board, 2397(1), 125-134.

http://dx.doi.org/10.3141/2397-15.

Lee, J. (2015). Impact of neighborhood walkability on trip generation and trip chaining: case of Los Angeles. Journal of Urban Planning and Development, 142(3), 05015013. http://dx.doi.org/10.1061/(ASCE)UP.19435444.0000312 .

Lee, J., He, S. Y., \& Sohn, D. W. (2017). Potential of converting short car trips to active trips: the role of the built environment in tour-based travel. Journal of Transport \& Health, 7, 134-148.

http://dx.doi.org/10.1016/j.jth.2017.08.008.

Lenzholzer, S. (2009). Engrained experience: a comparison of microclimate perception schemata and microclimate measurements in Dutch urban squares. International Journal of Biometeorology, 54(2), 141-150.

http://dx.doi.org/10.1007/s00484-009-0262-z. PMid:19760436.

Lerman, Y., \& Omer, I. (2016). Urban area types and spatial distribution of pedestrians: lessons from Tel Aviv. Computers, Environment and Urban Systems, 55, 11-23. http://dx.doi.org/10.1016/j.compenvurbsys.2015.09.010.

Lin, P., Gou, Z., Lau, S., \& Qin, H. (2017). The impact of urban design descriptors on outdoor thermal environment: a literature review. Energies, 10(12), 2151. http://dx.doi.org/10.3390/en10122151.

Lind, D. (2007). Zoning, urban form, and civic identity: the future of Pittsburgh's Hillsides Pittsburgh. Architectural Record, 195(5)

Littke, H. (2016). Revisiting the San Francisco parklets problematizing publicness, parks, and transferability. Urban Forestry \& Urban Greening, 15, 165-173. http://dx.doi.org/10.1016/j.ufug.2015.12.010.

Low, S. (2000). Cultura in the modern city: the microgeographies of gender, class, and generation in the costa rican plaza. Horizontes Antropológicos, 6(13), 31-64. http://dx.doi.org/10.1590/S0104-71832000000100003.

Low, S. (2009). Cerrando y reabriendo el espacio público en la ciudad latinoamericana. Cuadernos de Antropología Social, 30, 17-38.

Lubell, S. (2008). In a desert city, a skyline grows ever higher. Archtectural Record, 8.

Lunecke, M. G. H. (2016). Instrumentos de planificación y diseño urbano para promover al peatón en las ciudades chilenas. Un estudio comparado entre Chile y Alemania. Urbano, 19(34), 48-57. 
Lynch, K. (1960). The image of the city. Cambridge: The M.I.T. Press.

Maat, K., Van Wee, B., \& Stead, D. (2005). Land use and travel behaviour: expected effects from the perspective of utility theory and activity-based theories. Environment and Planning. B, Planning \& Design, 32(1), 33-46. http://dx.doi.org/10.1068/b31106.

Macdonald, E., \& Larice, M. (2013). The urban design reader (2nd ed.). Abingdon: Routledge.

Macedo, A. C. (2007). A Carta do Novo Urbanismo norte-americano. Arquitextos, 7(082.03). Recuperado em 20 de dezembro de 2018, de http://www.vitruvius.com.br/revistas/read/arquitextos/07.082/262

Madureira, A. M. (2015). Physical planning in place-making through design and image building. Journal of Housing and the Built Environment, 30(1), 157-172. http://dx.doi.org/10.1007/s10901-013-9381-2.

Mandanipour, A. (1999). Why are the design and development of public spaces significant for cities? Environment and Planning. B, Planning \& Design, 26(6), 879-891. http://dx.doi.org/10.1068/b260879.

Marcinkova, D. (2016). Greenery as art-design in urban public space. In Proceedings of the 3rd International Multidisciplinary Scientific Conference on Social Sciences and Arts SGEM2016 (pp. 359-366). New York: Curran Associates, Inc.

Marina, O. A., \& Velevski, S. (2014). Urban strategies for the future development of turin and skopje: the economic crisis and the effectiveness of urban design. In Proceedings of the Papers from the 9th Annual Conference on European Integration: The Europe of Tomorrow: Creative, Digital, Integrated (pp. 217-235). Skopje: AKS Management Skopje.

Matijosaitiene, I. (2016). Combination of CPTED and space syntax for the analysis of crime. Safer Communities, 15(1), 49-62. http://dx.doi.org/10.1108/SC-05-2015-0013.

Mehta, V. (2009). Look closely and you will see, listen carefully and you will hear: urban design and social interaction on streets. Journal of Urban Design, 14(1), 29-64. http://dx.doi.org/10.1080/13574800802452658.

Mehta, V. (2011). Small businesses and the vitality of main street. Journal of Architectural and Planning Research, 28(4), 271-291.

Melgar, O., \& Elena, M. (2015). El Palacio Municipal de Santiago de Cuba en la recuperación de la memoria colectiva. Arquitectura y Urbanismo, 36(2), 19-40.

Miles, M. (1996). Art and craft in urban design: the possibilities for collaboration. Urban Design International, 1(1), 81-88. http://dx.doi.org/10.1057/udi.1996.7.

Miles, M. (2002). After the public realm: spaces of representation, transition and plurality. Journal of Art \& Design Education, 19(3), 253-261. http://dx.doi.org/10.1111/1468-5949.00227.

Mills, G. (2005). Progress toward sustainable settlements: a role for urban climatology. Theoretical and Applied Climatology, 84, 69-76.

Mohamad zin, R., Ahmad, B., Keyvanfar, A., Shafaghat, A., Malik, T. A., Ahmad, M. H., Majid, M. A., Lamit, H., \& Yadollahi, M. (2013). Application of the Path Walkability Index (Pawdex) model: a case study of retail walking pattern recognition in Taman University Skudai, Johor, Malaysia. Advanced Science Letters, 19(10), 3021-3024.

Monteiro, A., Almeida, M., Velho, S., \& Fonseca, L. (2012). A (in)eficácia das políticas europeias e nacionais para prevenir os riscos causados pelas manifestações de mudança climática nos espaços urbanos. Revista da Faculdade de Letras, 1, 45-58. Recuperado em 15 de julho de 2018, de https://hdl.handle.net/10216/83127

Monteiro, A., Almeida, M., Velho, S., \& Fonseca, L. (2013). A (in)eficácia das políticas europeias e nacionais para prevenir os riscos causados pelas manifestações de mudança climática nos espaços urbanos. Revista da Faculdade de Letras - Geografia, 2, 97-120.

Moraes, V., No., \& Saboya, R. T. (2010). A urgência do planejamento: a revisão dos instrumentos normativos de ocupação urbana. Arquitextos, 11(125.02). Recuperado em 10 de junho de 2018, de http://www.vitruvius.com.br/revistas/read/arquitextos/10.125/3624 
Moulay, A., \& Ujang, N. (2016). Legibility of neighborhood parks and its impact on social interaction in a planned residential area. Archnet-IJAR, 10(1), 184-194. http://dx.doi.org/10.26687/archnet-ijar.v10i1.686.

Muhs, C. D., \& Cliffon, K. J. (2016). Do characteristics of walkable environments support bicycling? Toward a definition of bicycle-supported development. Journal of Transport and Land Use, 9(2), 147-188.

Mukhija, V., \& Shoup, D. (2006). Quantity versus quality in off-street parking requirements. Journal of the American Planning Association, 72(3), 72. http://dx.doi.org/10.1080/01944360608976752.

Narayanan, Y. (2012). Violence against women in delhi: a sustainability problematic. Journal of South Asian Development, 7(1), 1-22. http://dx.doi.org/10.1177/097317411200700101.

Nikolaeva, A. (2012). Designing public space for mobility: contestation, negotiation and experiment at Amsterdam airport schiphol. Tijdschrift voor Economische en Sociale Geografie, 103(5), 542-554.

http://dx.doi.org/10.1111/j.1467-9663.2012.00740.x.

Nobre, E. A. C. (2006). O ideário urbanístico e a legislação na cidade de São Paulo. In Anais do IX Seminário de História da Cidade e do Urbanismo. São Paulo: FAUUSP/FAUMACK/PUCCAMP/EESC.

Noto, F. S. (2017). O quarteirão como suporte da transformação (Tese de doutorado). Faculdade de Arquitetura e Urbanismo, Universidade de São Paulo, São Paulo.

Nunes, R. T. S., Prodanoff, J. H. A., Nunes, B., \& Freitas, M. A. V. (2011). Incorporating Water Sensitive Urban Design (WSUD) practices into the planning contexto: the conceptual case for lot-scale developments. Sustainability Today, 167, 341-352. http://dx.doi.org/10.2495/ST110311.

Nyka, L. (2016). Polder and city: sustaining water landscapes on an urban edge. In Proceedings of the Conference Proceedings of International Multidisciplinary Scientific Conferences on Social Sciences and Arts SGEM2016 (pp. $493-$ 500). New York: Curran Associates, Inc.

Oc, T., \& Tiesdell, S. (1999). The Fortress, the panoptic, the regulatory and the animated: planning and urban design approaches to safer city centres. Landscape Research, 24(3), 265-286.

http://dx.doi.org/10.1080/01426399908706563.

Oktay, D. (2017). Reevaluating urban identity under changing circumstances: the case of Samsun, Turkey. Proceedings of the Institution of Civil Engineers. Urban Design and Planning, 170(5), 189-204. http://dx.doi.org/10.1680/jurdp.17.00001.

Olaru, D., Smith, B., \& Taplin, J. (2011). Residential location and transit-oriented development in a new rail corridor. Transportation Research Part A, Policy and Practice, 45(3), 219-237.

http://dx.doi.org/10.1016/j.tra.2010.12.007.

Online-Utility.org. (2018). Text analyzer. Recuperado em 27 de dezembro de 2018, de https://www.onlineutility.org/text/analyzer.jsp

Oranratmanee, R., \& Sachakul, V. (2014). Streets as public spaces in southeast Asia: case studies of thai pedestrian streets. Journal of Urban Design, 19(2), 211-229. http://dx.doi.org/10.1080/13574809.2013.870465.

Özbil, A., Yesiltepe, D., \& Argin, G. (2015). Modeling walkability: the effects of street design, street-network configuration and land-use on pedestrian movement. A/Z ITU Journal of the Faculty of Architecture, 12, $189-207$.

Özer, Ö., \& Kubat, A. S. (2014). Walkability: perceived and measured qualities in action. A/Z ITU Journal of the Faculty of Architecture, 11, 101-117.

Özer, Ö., \& Kubat, A. S. (2015). Measuring walkability in istanbul galata region. A/Z ITU Journal of the Faculty of Architecture, 12, 15-29.

Özgür, E. F. (2013). Urban design projects and the planning process: the kadiköy old market area revitalization project and the kartal industrial area regeneration project. Cities (London, England), 31, 208-219.

http://dx.doi.org/10.1016/j.cities.2012.05.003. 
Palermo, C., \& Ponzini, D. (2012). At the crossroads between urban planning and urban design: critical lessons from Three Italian case studies. Planning Theory \& Practice, 13(3), 1-16.

http://dx.doi.org/10.1080/14649357.2012.701661.

Pan, H., Shen, Q., \& Zhang, M. (2009). Influence of urban form on travel behaviour in four neighbourhoods of Shanghai. Urban Studies (Edinburgh, Scotland), 46(2), 275-294. http://dx.doi.org/10.1177/0042098008099355.

Papagiannakis, A., \& Vitopoulou, A. (2015). An urban strategy in time of crisis: mobility management and low-cost public space design. Spatium (Belgrade), 33(33), 1-7. http://dx.doi.org/10.2298/SPAT1533001P.

Parvin, A., Min, Ye., \& Beisi, J. (2008). Effect of visibility on multilevel movement: a study of the high-density compact built environment in Hong Kong. Urban Design International, 13(3), 169-181.

http://dx.doi.org/10.1057/udi.2008.22.

Patel, S. B. (2011). Analyzing urban layouts - can high density be achieved with good living conditions? Environment and Urbanization, 23(2), 583-595. http://dx.doi.org/10.1177/0956247811418737.

Payton, N. I., \& Hawkes, A. (2013). Designing new transit systems using a transect-based model. Proceedings of the Institution of Civil Engineers - Urban Design and Planning, 166(4), 217-228.

https://doi.org/10.1680/udap.11.00003.

Penn, A., Hillier, B., Banister, D., \& Xu, J. (1998). Configurational modelling of urban movement networks. Environment and Planning. B, Planning \& Design, 25(1), 59-84. http://dx.doi.org/10.1068/b250059.

Pérez Lancellotti, G. (2014). The master plan as a tool for urban design: potentials and restrictions. The case of Antofagasta. Revista AUS, 15, 16-21.

Pickett, S. T. A., \& Cadenasso, M. L. (2007). Linking ecological and built components of urban mosaics: an open cycle of ecological design. Journal of Ecology, 96(0), 8-12. http://dx.doi.org/10.1111/j.1365-2745.2007.01310.x.

Pinto, A. J., \& Brandão, A. (2015). A multi-scale approach of public space networks in the scattered city. URBAN DESIGN International, 20(3), 175-194. http://dx.doi.org/10.1057/udi.2015.4.

Pizarro, R. E. (2009). Teaching to understand the urban sensorium in the digital age: lessons from the studio. Design Studies, 30(3), 272-286. http://dx.doi.org/10.1016/j.destud.2008.09.002.

Pour Ebrahim, N. (2015). Sense of community in new urbanism neighbourhoods: a review. Open House International, 40(4), 25-29.

Pucher, J., \& Buehler, R. (2010). Walking and cycling for healthy cities. Built Environment, 36(4), 391-414. http://dx.doi.org/10.2148/benv.36.4.391.

Punter, J. (2002). Urban design as public policy: evaluating the design dimension of Vancouver's planning system. International Planning Studies, 7(4), 265-282. http://dx.doi.org/10.1080/1356347022000027710.

Punter, J. (2007). Developing urban design as public policy: best practice principles for design review and development management. Journal of Urban Design, 12(2), 167-202.

http://dx.doi.org/10.1080/13574800701306195.

Raeisi, I., Kharazmi Nezhad, A., \& Hafezifar, M. (2010). Architectural design principles of public spaces based on social sustainability approach: a case study in Ardabil, Iran. Design Principles \& Practices, 4(5), 99-113. http://dx.doi.org/10.18848/1833-1874/CGP/v04i05/37968.

Ragheb, A. A., El-Darwish, I. I., \& Ahmed, S. (2016). Microclimate and human comfort considerations in planning a historic urban quarter. International Journal of Sustainable Built Environment, 5(1), 156-167.

https://doi.org/10.1016/j.ijsbe.2016.03.00.

Raman, S. (2010). Designing a liveable compact city: physical forms of city and social life in urban neighbourhoods. Built Environment, 36(1), 63-80. http://dx.doi.org/10.2148/benv.36.1.63.

Rodríguez Algeciras, J. A., Coch, H., De la Paz Pérez, G., Chaos Yeras, M., \& Matzarakis, A. (2015). Human thermal comfort conditions and urban planning in hot-humid climates - the case of Cuba. International Journal of Biometeorology, 60(80), 1151-1164. PMid:26628421. 
Rodríguez, M. I., Cuevas, M. M., Martínez, G., \& Moreno, B. (2014). Planning criteria for water sensitive urban design. WIT Transactions on Ecology and the Environment, 191, 1579-1591. http://dx.doi.org/10.2495/SC141342.

Rodríguez-Rojas, M. I., Cuevas-Arrabal, M. M., Escobar, B. M., \& Montes, G. M. (2017). The paradigm change of urban drainage management from the planning perspective. A methodological proposal. Boletín de la Asociación de Geógrafos Españoles, 75, 55-74.

Rotmeyer, J. (2006). Can elevated pedestrian walkways be sustainable? WIT Transactions on Ecology and the Environment, 93, 293-302. http://dx.doi.org/10.2495/SC060281.

Ruban, L. (2016). Natural-hydrological protection for waterfront territories: "blue-green" initiative. In Proceedings of the Conference International Multidisciplinary Scientific Conferences on Social Sciences and Arts SGEM2016 (pp. 443-450). New York: Curran Associates, Inc.

Santos Nouri, A. (2015). A framework of thermal sensitive urban design benchmarks: potentiating the longevity of auckland's public realm. Journal of Buildings, 5(1), 252-281. http://dx.doi.org/10.3390/buildings5010252.

Santos Nouri, A., \& Costa, J. P. (2017). Placemaking and climate change adaptation: new qualitative and quantitative considerations for the "Place Diagram". Journal of Urbanism, 10(3), 1-27.

http://dx.doi.org/10.1080/17549175.2017.1295096.

São Paulo. Prefeitura Municipal. (2014, 31 de julho). Lei no 16.050, de 31 de julho de 2014: Aprova a Política de Desenvolvimento Urbano e o Plano Diretor Estratégico do Município de São Paulo e revoga a Lei no 13.430/2002. São Paulo: Diário Oficial da Cidade.

São Paulo. Prefeitura Municipal. (2016, 22 de março). Lei no 16.402, de 22 de março de 2016: Disciplina o parcelamento, o uso e a ocupação do solo no Município de São Paulo, de acordo com a Lei no 16.050, de 31 de julho de 2014 - Plano Diretor Estratégico (PDE). São Paulo: Diário Oficial da Cidade e.

Schaefer, V., \& Higgs, E. (2007). Modern babylon. Alternatives Journal, 33, 26-29.

Schmitt, S.-M., \& Hartmann, T. (2016). Clumsy city by design: a theory for Jane Jacobs' imperfect cities? Urban Planning, 1(4), 2183-7635. http://dx.doi.org/10.17645/up.v1i4.732.

Shapiro, A. (2016). The mezzanine. Space and Culture, 19(4), 292-307.

http://dx.doi.org/10.1177/1206331216631294.

Sholihah, A. (2017). Street image of traditional street: the case of Pecinan street, Magelang, Indonesia. Journal of Engineering and Applied Sciences (Asian Research Publishing Network), 12, 3381-3386.

Shoup, D. (1997). The high cost of free parking. Journal of Planning Education and Research, 17(1), 3-20.

http://dx.doi.org/10.1177/0739456X9701700102.

Shoup, D. (1999). In lieu of required parking. Journal of Planning Education and Research, 18(4), 307-320. http://dx.doi.org/10.1177/0739456X9901800403.

Shrestha, B. K. (2011). Street typology in Kathmandu and street transformation. Urbani Izziv, 22(2), 107-121. http://dx.doi.org/10.5379/urbani-izziv-en-2011-22-02-004.

Sillano, M., Greene, M., \& Ortúzar, J. D. (2006). Cuantificando la percepción de inseguridad ciudadana en barrios de escasos recursos. EURE (Santiago), 32(97), 17-35.

Silva, J. C., \& Souza, C. A. (2016). The urban question in the upper paraguay basin: urban development and its implications in the drainage canals in Cáceres - MT. Boletim de Geografia, 34(3), 111-128.

http://dx.doi.org/10.4025/bolgeogr.v34i3.22360.

Silva, J. M. P. (2011). Social housing and the municipal laws from campinas metropolitan region. Ambiente Construído, 11(3), 55-71. http://dx.doi.org/10.1590/S1678-86212011000300005.

Sinemillioglu, M. O., Akın, C., \& Karacay, N. (2010). Relationship between green areas and urban conservation in historical areas and its reflections: case of Diyarbakir city, Turkey. European Planning Studies, 18(5), 775-789. http://dx.doi.org/10.1080/09654311003612620. 
Siqueira-Gay, J., Dibo, A. P. A., \& Giannotti, M. A. (2017). Vulnerabilidade as ilhas de calor no municipio de São Paulo: uma abordagem para a implantacao de medidas mitigadoras na gestao urbana. Revista de Gestão Ambiental e da Sustentabilidade, 6(2), 105-123. http://dx.doi.org/10.5585/geas.v6i2.902.

Sorensen, A. (2006). Liveable cities in Japan: population ageing and decline as vectors of change. International Planning Studies, 11(3-4), 225-242. http://dx.doi.org/10.1080/13563470701231703.

Southworth, B. (2003). Urban design in action: the city of Cape Town's Dignified Places Programme Implementation of new public spaces towards integration and urban regeneration in South Africa. URBAN DESIGN International, 8(3), 119-133. http://dx.doi.org/10.1057/palgrave.udi.9000097.

Southworth, M., \& Parthasarathy, B. (1996). The suburban public realm I: its emergence, growth and transformation in the American metropolis. Journal of Urban Design, 1(3), 245-264.

http://dx.doi.org/10.1080/13574809608724385.

Speck, J. (2016). Cidade caminhável. São Paulo: Perspectiva.

Speranza, P., \& Viader, M. (2015). High resolution air quality and urban design. International Journal of Design Sciences and Technology, 21, 133-151.

Sternberg, E. (2002). What makes buildings catalytic? How cultural facilities can be designed to spur surrounding development. Journal of Architectural and Planning Research, 19, 30-43.

Sterrett, K., Hackett, M., \& Hill, D. (2012). The social consequences of broken urban structures: a case study of Belfast. Journal of Transport Geography, 21, 49-61. http://dx.doi.org/10.1016/j.jtrangeo.2012.01.014.

Stummvoll, G. (2004). Design against crime in vienna: a feminist approach. Crime Prevention and Community Safety: an International Journal, 6(4), 71-82. http://dx.doi.org/10.1057/palgrave.cpcs.8140202.

Sung, H., \& Oh, J.-T. (2011). Transit-oriented development in a high-density city: identifying its association with transit ridership in Seoul, Korea. Cities (London, England), 28(1), 70-82.

http://dx.doi.org/10.1016/j.cities.2010.09.004.

Swapan, A. Y. (2016). Holistic strategy for urban design: a microclimatic concern to sustainability. Journal of Urban and Environmental Engineering, 10(2), 221-232.

Taylor, L., \& Hochuli, D. (2015). Creating better cities: how biodiversity and ecosystem functioning enhance urban residents' wellbeing. Urban Ecosystems, 18(3), 1-16. http://dx.doi.org/10.1007/s11252-014-0427-3.

Tilaki, M. J. M., Aldrin, A., Bahauddin, A., \& Marzbali, H. M. (2013). The influence of landuse planning on the making of the cities physical identity in Iran: an analytical review and commentary. Middle East Journal of Scientific Research, 14, 762-772. http://dx.doi.org/10.5829/idosi.mejsr.2013.14.6.685.

Tomich, S. (2002). Genius loci: a poetic approach to urban design. Plan Canada, 42(3), 32034.

Tork, L. D., Tibiriçá, A. C. G., \& Tibiriçá, Á. M. B. (2017). Análise da ventilação natural conforme planos diretores: resultados de pesquisa em Belém, PA. Ambiente Construído, 17(1), 329-351. https://dx.doi.org/10.1590/s167886212017000100138.

Turkienicz, B. (Org.) (1984). Desenho urbano. In Anais do I SEDUR - Seminário sobre Desenho Urbano no Brasil (Vol. 1-3). São Paulo: Projeto Editores.

Turkienicz, B., \& Malta, M. (Org.) (1986). Desenho urbano. In Anais do II SEDUR - Seminário sobre Desenho Urbano no Brasil. São Paulo: CNPq/Finep/Pini.

Urrutiaguer, M., Lloyd, S., \& Lamshed, S. (2010). Determining water sensitive urban design project benefits using a multi-criteria assessment tool. Water Science and Technology: a Journal of the International Association on Water Pollution Research, 61, 2333-2341.

Vale, D. (2015). Transit-oriented development, integration of land use and transport, and pedestrian accessibility: combining node-place model with pedestrian shed ratio to evaluate and classify station areas in Lisbon. Journal of Transport Geography, 45, 70-80. http://dx.doi.org/10.1016/j.jtrangeo.2015.04.009. 
Van der Spek, S. C., Van Langelaar, C. M., \& Kickert, C. C. (2013). Evidence-based design: satellite positioning studies of city centre user groups. Urban Design and Planning, 166(4), 206-2016.

http://dx.doi.org/10.1680/udap.11.00028.

Van Deusen, R., Jr. (2002). Public space design as class warfare: urban design, the 'right to the city' and the production of Clinton Square, Syracuse, NY. GeoJournal, 58(2/3), 149-158.

http://dx.doi.org/10.1023/B:GEJ0.0000010834.17907.5e.

Vanti, N. A. P. (2002). Da bibliometria à webometria: uma exploração conceitual dos mecanismos utilizados para medir o registro da informação e a difusão do conhecimento. Ciência da Informação, 31(2), 152-162. http://dx.doi.org/10.1590/S0100-19652002000200016.

Vasconcelos, A. F., Miguez, M. G., \& Vazquez, E. G. (2016). Critérios de projeto e benefícios esperados da implantação de técnicas compensatórias em drenagem urbana para controle de escoamentos na fonte, com base em modelagem computacional aplicada a um estudo de caso na zona oeste do Rio de Janeiro. Engenharia Sanitaria e Ambiental, 21(4), 655-662. http://dx.doi.org/10.1590/s1413-41522016146469.

Walford, N., Phillips, J., Hockey, A., \& Pratt, S. (2017). Assessing the needs of older people in urban settings: integration of emotive, physiological and built environment data. Geo: Geography and Environment, 4(1), 1-20. http://dx.doi.org/10.1002/geo2.37.

Weinberger, R. (2014). Three faces of parking: emerging trends in the U.S. Transport and Sustainability, 5, $235-258$. http://dx.doi.org/10.1108/S2044-994120140000005021.

Wey, W. M. (2011). A study of the built environment design elements embedded into the multiple criteria strategic planning model for an urban renewal. International Journal of civil and Evironmental Engineering, 5(4), $157-167$. https://doi.org/10.1999/1307-6892/1800.

Williams, L. (2001). Reshaping Melbourne. Art and Australia, 38(2), 326-328.

Wood, L., Frank, L. D., \& Giles-corti, B. (2010). Sense of community and its relationship with walking and neighborhood design. Social Science \& Medicine, 70(9), 1381-1390.

http://dx.doi.org/10.1016/j.socscimed.2010.01.021. PMid:20189699.

Woodcock, I., Dovey, K., Wollan, S., \& Beyerle, A. (2010). Modelling the compact city: capacities and Visions for Melbourne. Australian Plants, 47(2), 94-104. http://dx.doi.org/10.1080/07293681003767793.

Wright, L. (2001). Latin American Busways: moving people rather than cars. Natural Resources Forum, 25(2), 121134. http://dx.doi.org/10.1111/j.1477-8947.2001.tb00754.x.

Xinxin, Z., \& Jie, Z. (2017). Study on urban design of luohu railway station area from the perspective of integrated urban design. Journal of Landscape Research, 9(3), 47-56.

Yahia, M., \& Johansson, E. (2014). Landscape interventions in improving thermal comfort in the hot dry city of Damascus, Syria-The example of residential spaces with detached buildings. Landscape and Urban Planning, 125, 125. http://dx.doi.org/10.1016/j.landurbplan.2014.01.014.

Yiannakou, A., \& Salata, K. (2017). Adaptation to climate change through spatial planning in compact urban areas: a case study in the city of Thessaloniki. Sustainability, 9(2), 9. http://dx.doi.org/10.3390/su9020271.

Yuan, C., Norford, L., Britter, R., \& Ng, E. (2016). A modelling-mapping approach for fine-scale assessment of pedestrian-level wind in high-density cities. Building and Environment, 97, 152-165.

http://dx.doi.org/10.1016/j.buildenv.2015.12.006.

Zacharias, J., Tianxin, Z., \& Nakajima, N. (2011). Tokyo Station City: the railway station as urban place. URBAN DESIGN International, 16(4), 242-251. http://dx.doi.org/10.1057/udi.2011.15.

Zakaria, R., Munikanan, V., Said, M., Saleh, A. L., Wah, C., Shakri, A., \& Rajuna, M. (2012). Sustainable Neighbourhood Elements Model (SNEs Model). Advanced Science Letters, 15(1), 113-116.

http://dx.doi.org/10.1166/asl.2012.4029.

\section{Editor: Fábio Duarte.}

Recebido: Jul. 17, 2018

Aprovado: Mar. 13, 2019 
Apêndice A. Artigos selecionados através da RSL que relacionam desenho urbano e espaço público

\begin{tabular}{|c|c|c|}
\hline ID & Autor/Ano & Título do artigo \\
\hline A & Boulange et al. (2017) & $\begin{array}{l}\text { Examining associations between urban design attributes and transport mode } \\
\text { choice for walking, cycling, public transport and private motor vehicle trips }\end{array}$ \\
\hline A & Lee et al. (2017) & $\begin{array}{l}\text { Potential of converting short car trips to active trips: The role of the built } \\
\text { environment in tour-based travel }\end{array}$ \\
\hline A & Huang et al. (2017) & $\begin{array}{l}\text { Travel analytics: Understanding how destination choice and business clusters are } \\
\text { connected based on social media data }\end{array}$ \\
\hline A & Dovey \& Pafka (2017) & What is functional mix? An assemblage approach \\
\hline A & Gunn et al. (2017) & $\begin{array}{l}\text { Designing healthy communities: Creating evidence on metrics for built } \\
\text { environment features associated with walkable neighbourhood activity } \\
\text { centres }\end{array}$ \\
\hline A & Balsas (2017) & The right to walk in cities, a comparative review of Macau, Lisbon and Las Vegas \\
\hline A & Goins et al. (2016) & Local Health Department Engagement in Community Physical Activity Policy \\
\hline A & Muhs \& Cliffon (2016) & $\begin{array}{l}\text { Do characteristics of walkable environments support bicycling? Toward a } \\
\text { definition of bicycle-supported development }\end{array}$ \\
\hline A & Lee (2015) & $\begin{array}{l}\text { Impact of neighborhood walkability on trip generation and trip chaining: Case of } \\
\text { Los Angeles }\end{array}$ \\
\hline A & Lerman \& Omer (2016) & Urban area types and spatial distribution of pedestrians: Lessons from Tel Aviv \\
\hline A & Koohsari et al. (2016) & $\begin{array}{l}\text { Walkability and walking for transport: characterizing the built environment using } \\
\text { space syntax }\end{array}$ \\
\hline A & Lunecke (2016) & $\begin{array}{l}\text { Instrumentos de planificación y diseño urbano para promover al peatón en las } \\
\text { ciudades chilenas. Un estudio comparado entre Chile y Alemania }\end{array}$ \\
\hline A & Ewing (2016) & Contribution of urban design qualities to pedestrian activity \\
\hline A & $\begin{array}{l}\text { Papagiannakis \& } \\
\text { Vitopoulou (2015) }\end{array}$ & $\begin{array}{l}\text { An urban strategy in time of crisis: Mobility management and low-cost public } \\
\text { space design }\end{array}$ \\
\hline A & Özer \& Kubat (2015) & Measuring walkability in Istanbul Galata region \\
\hline A & Özbil et al. (2015) & $\begin{array}{l}\text { Modeling walkability: The effects of street design, street-network configuration and } \\
\text { land-use on pedestrian movement }\end{array}$ \\
\hline A & Atkinson (2015) & A Thick Green Line: Extracting Urban Space from Vehicle Infrastructure \\
\hline A & Aydin \& Ayataç (2015) & $\begin{array}{l}\text { A pedesterianization case in the context of public interest: A pedesterianization } \\
\text { project in Eminönü historical peninsula }\end{array}$ \\
\hline A & Fernandez Mucoz (2015) & The experience of urban walking as a guidance for urban quality assessment \\
\hline A & $\begin{array}{l}\text { Oranratmanee \& } \\
\text { Sachakul (2014) }\end{array}$ & Streets as Public Spaces in Southeast Asia: Case Studies of Thai Pedestrian Streets \\
\hline A & Choi (2014) & Walkability and the complexity of walking behavior \\
\hline A & Özer \& Kubat (2014) & Walkability: Perceived and measured qualities in action \\
\hline A & $\begin{array}{l}\text { Mohamad zin et al. } \\
\text { (2013) }\end{array}$ & $\begin{array}{l}\text { Application of the Path Walkability Index (Pawdex) model: A case study of retail } \\
\text { walking pattern recognition in Taman University Skudai, Johor, Malaysia }\end{array}$ \\
\hline A & $\begin{array}{l}\text { Djukic \& Vukmirovic } \\
\text { (2012) }\end{array}$ & $\begin{array}{l}\text { Redesigning the network of pedestrian Spaces in the function of reduction of CO2 } \\
\text { Emission. Case study: Pančevo and Vršac }\end{array}$ \\
\hline A & Appleyard (2012) & Sustainable and healthy travel choices and the built environment \\
\hline A & Asl et al. (2012) & $\begin{array}{l}\text { The role of pedestrian streets in sustainability of urban spaces, case study: Tabriz } \\
\text { Tarbiyat Street, Iran }\end{array}$ \\
\hline A & Shrestha (2011) & Street typology in Kathmandu and street transformation \\
\hline A & Boarnet et al. (2011) & $\begin{array}{l}\text { The street level built environment and physical activity and walking: Results of a } \\
\text { predictive validity study for the irvine minnesota inventory }\end{array}$ \\
\hline A & Wood et al. (2010) & Sense of community and its relationship with walking and neighborhood design \\
\hline A & Ewing \& Cervero (2001) & Travel and the Built Environment: A Synthesis \\
\hline A & Pucher \& Buehler (2010) & Walking and cycling for healthy cities \\
\hline A & Crane (2010) & The Influence of Urban Form on Travel: An Interpretive Review \\
\hline A & Pan et al. (2009) & Influence of urban form on travel behaviour in four neighbourhoods of Shanghai \\
\hline A & $\begin{array}{l}\text { Kemperman \& } \\
\text { Timmermans (2009) }\end{array}$ & $\begin{array}{l}\text { Influences of built environment on walking and cycling by latent segments of } \\
\text { aging population }\end{array}$ \\
\hline A & Mehta (2009) & $\begin{array}{l}\text { Look Closely and You Will See, Listen Carefully and You Will Hear: Urban Design } \\
\text { and Social Interaction on Streets }\end{array}$ \\
\hline A & Johnson \& Marko (2008) & Designing Healthy Places: Land use planning and public health \\
\hline A & Parvin et al. (2008) & $\begin{array}{l}\text { Effect of visibility on multilevel movement: A study of the high-density compact } \\
\text { built environment in Hong Kong }\end{array}$ \\
\hline
\end{tabular}




\begin{tabular}{|c|c|c|}
\hline ID & Autor/Ano & Título do artigo \\
\hline A & Badland et al. (2008) & $\begin{array}{l}\text { Travel behavior and objectively measured urban design variables: Associations for } \\
\text { adults traveling to work }\end{array}$ \\
\hline A & Kenworthy (2007) & Urban Planning and Transport Paradigm Shifts for Cities of the Post-Petroleum Age \\
\hline A & Rotmeyer (2006) & Can elevated pedestrian walkways be sustainable? \\
\hline A & Maat et al. (2005) & $\begin{array}{l}\text { Land use and travel behaviour: Expected effects from the perspective of utility } \\
\text { theory and activity-based theories }\end{array}$ \\
\hline A & $\begin{array}{l}\text { Badland \& Schofield } \\
(2005)\end{array}$ & Transport, urban design, and physical activity: an evidence-based update \\
\hline A & Hamilton-Baillie (2004) & $\begin{array}{l}\text { Urban design: Why don't we do it in the road? Modifying traffic behavior through } \\
\text { legible urban design }\end{array}$ \\
\hline A & Krizek (2003) & $\begin{array}{l}\text { Operationalizing Neighborhood Accessibility for Land Use:Travel Behavior } \\
\text { Research and Regional Modeling }\end{array}$ \\
\hline A & Greenwald (2003) & $\begin{array}{l}\text { The Road Less Traveled: New Urbanist Inducements to Travel Mode Substitution for } \\
\text { Nonwork Trips }\end{array}$ \\
\hline A & Badiozamani (2003) & Car-free days: A shift in the planning paradigm? \\
\hline A & Cervero (2002) & Built environments and mode choice: Toward a normative framework \\
\hline A & Handy et al. (2002) & How the built environment affects physical activity: Views from urban planning \\
\hline A & Boarnet \& Crane (2001) & $\begin{array}{l}\text { The influence of land use on travel behavior: Specification and estimation } \\
\text { strategies }\end{array}$ \\
\hline A & Penn et al. (1998) & Configurational Modelling of Urban Movement Networks \\
\hline A & Abdel-Salam (1996) & $\begin{array}{l}\text { Perception of qualitative changes in public open spaces: The case of Alexandria, } \\
\text { Egypt }\end{array}$ \\
\hline B & Anderson et al. (2016) & $\begin{array}{l}\text { Lively Social Space, Well-Being Activity, and Urban Design: Findings From a Low- } \\
\text { Cost Community-Led Public Space Intervention }\end{array}$ \\
\hline B & Giraldo (2015) & $\begin{array}{l}\text { Producción social, proceso participativo e intervención sostenible en el espacio } \\
\text { público de los centros históricos: el caso de Pamplona, Colombia }\end{array}$ \\
\hline B & Elsheshtawy (2015) & Observing the public realm: William whytes the social life of small urban spaces \\
\hline B & Pinto \& Brandão (2015) & A multi-scale approach of public space networks in the scattered city \\
\hline B & Dymitrow (2014) & $\begin{array}{l}\text { The effigy of urbanity or a rural parody? A visual approach to small-town public } \\
\text { space }\end{array}$ \\
\hline B & $\begin{array}{l}\text { Van der Spek et al. } \\
(2013)\end{array}$ & Evidence-based design: Satellite positioning studies of city centre user groups \\
\hline B & Habib et al. (2013) & $\begin{array}{l}\text { Urban deteriorated fabric regeneration according to public open space } \\
\text { enhancement }\end{array}$ \\
\hline B & Giddings et al. (2011) & Public squares in European city centres \\
\hline B & Aelbrecht (2010) & Rethinking urban design for a changing public life \\
\hline B & Haas (2009) & $\begin{array}{l}\text { Traditional European squares in contemporary urbanism: Dubrovnik's medieval } \\
\text { squares }\end{array}$ \\
\hline B & Alves et al. (2009) & Getting urban profits through the freedom of use \\
\hline B & Low (2009) & Cerrando y reabriendo el espacio público en la ciudad latinoame \\
\hline B & Frick (2007) & Spatial synergy and supportiveness of public space \\
\hline B & Balsas (2007) & City centre revitalization in Portugal: A study of Lisbon and Porto \\
\hline B & Jackson (2003) & The relationship of urban design to human health and condition \\
\hline B & Biddulph (1999) & Bringing vitality to a campus environment \\
\hline B & $\begin{array}{l}\text { Southworth \& } \\
\text { Parthasarathy (1996) }\end{array}$ & $\begin{array}{l}\text { The suburban public realm I: its emergence, growth and transformation in the } \\
\text { American metropolis }\end{array}$ \\
\hline C & Cárdenas O'Byrne (2017) & Medir el uso del espacio público urbano seguro \\
\hline C & $\begin{array}{l}\text { Cárdenas O'Byrne \& } \\
\text { Verónica D'Inca (2016) }\end{array}$ & $\begin{array}{l}\text { Arquitectura de la noviolencia: el papel del otro en la construcción del espacio } \\
\text { común }\end{array}$ \\
\hline C & Matijosaitiene (2016) & Combination of CPTED and space syntax for the analysis of crime \\
\hline C & Narayanan (2012) & Violence Against Women in Delhi: A Sustainability Problematic \\
\hline C & Davis (2013) & $\begin{array}{l}\text { Zero-Tolerance Policing, Stealth Real Estate Development, and the Transformation } \\
\text { of Public Space: Evidence from Mexico City }\end{array}$ \\
\hline C & Mehta (2011) & Small businesses and the vitality of main street \\
\hline C & Sillano et al. (2006) & $\begin{array}{l}\text { Cuantificando la Percepción de Inseguridad Ciudadana en Barrios de Escasos } \\
\text { Recursos }\end{array}$ \\
\hline C & Stummvoll (2004) & Design against crime in vienna: A feminist approach \\
\hline C & Oc \& Tiesdell & $\begin{array}{l}\text { The fortress, the panoptic, the regulatory and the animated: Planning and urban } \\
\text { design approaches to safer city centres }\end{array}$ \\
\hline
\end{tabular}




\begin{tabular}{|c|c|c|}
\hline ID & Autor/Ano & Título do artigo \\
\hline C & Doeksen (1997) & $\begin{array}{l}\text { Reducing crime and the fear of crime by reclaiming New Zealand's suburban } \\
\text { street }\end{array}$ \\
\hline $\mathrm{D}$ & $\begin{array}{l}\text { Aguilera-Martínez, et al. } \\
\text { (2017) }\end{array}$ & $\begin{array}{l}\text { Intervención social en el borde urbano desde el proceso de la significación } \\
\text { cultural }\end{array}$ \\
\hline $\mathrm{D}$ & Sholihah (2017) & Street image of traditional street: The case of Pecinan street, Magelang, Indonesia \\
\hline $\mathrm{D}$ & Al-Bishawi et al. (2015) & $\begin{array}{l}\text { Women's behaviour in public spaces and the influence of privacy as a cultural } \\
\text { value: The case of Nablus, Palestine }\end{array}$ \\
\hline $\mathrm{D}$ & Oktay (2017) & $\begin{array}{l}\text { Reevaluating urban identity under changing circumstances: The case of Samsun, } \\
\text { Turkey }\end{array}$ \\
\hline $\mathrm{D}$ & Melgar \& Elena (2015) & $\begin{array}{l}\text { El Palacio Municipal de Santiago de Cuba en la recuperación de la memoria } \\
\text { colectiva }\end{array}$ \\
\hline $\mathrm{D}$ & Pizarro (2009) & $\begin{array}{l}\text { Teaching to understand the urban sensorium in the digital age: lessons from the } \\
\text { studio }\end{array}$ \\
\hline $\mathrm{D}$ & Tomich (2002) & Genius loci: A poetic approach to urban design \\
\hline $\mathrm{D}$ & Miles (2002) & After the public realm: Spaces of representation, transition and plurality \\
\hline $\mathrm{D}$ & Low (2000) & $\begin{array}{l}\text { Cultura in the modern city: the microgeographies of gender, class, and } \\
\text { generation in the costa rican plaza }\end{array}$ \\
\hline $\mathrm{E}$ & Heffernan et al. (2014) & $\begin{array}{l}\text { The relationship between the quality of active frontages and public perceptions of } \\
\text { public spaces }\end{array}$ \\
\hline $\mathrm{E}$ & Allweil \& Kallus (2013) & $\begin{array}{l}\text { Re-forming the political body in the city: The interplay of male bodies and territory } \\
\text { in urban public spaces in Tel Aviv }\end{array}$ \\
\hline $\mathrm{E}$ & Sterrett et al. (2012) & The social consequences of broken urban structures: A case study of Belfast \\
\hline $\mathrm{E}$ & Van Deusen (2002) & $\begin{array}{l}\text { Public space design as class warfare: Urban design, the 'right to the city' and the } \\
\text { production of Clinton Square, Syracuse, NY }\end{array}$ \\
\hline $\mathrm{E}$ & Mandanipour (1999) & Why are the design and development of public spaces significant for cities? \\
\hline $\mathrm{F}$ & Marcinkova (2016) & Greenery as art-design in urban public space \\
\hline $\mathrm{F}$ & $\begin{array}{l}\text { Dukanovic \& Zivkovic } \\
(2015)\end{array}$ & Public art \& public space programme: Learning, but doing! \\
\hline $\mathrm{F}$ & Williams (2001) & Reshaping Melbourne \\
\hline $\mathrm{F}$ & Miles (1996) & Art and craft in urban design - The possibilities for collaboration \\
\hline G & Chong et al. (2015) & $\begin{array}{l}\text { Psychosocial Well-Being of the Elderly and Their Perception of Matured Estate in } \\
\text { Singapore }\end{array}$ \\
\hline G & Walford et al. (2017) & $\begin{array}{l}\text { Assessing the needs of older people in urban settings: integration of emotive, } \\
\text { physiological and built environment data }\end{array}$ \\
\hline G & Sorensen (2006) & Liveable Cities in Japan: Population ageing and decline as Vectors of change \\
\hline $\mathrm{H}$ & $\begin{array}{l}\text { Schmitt \& Hartmann } \\
(2016)\end{array}$ & Clumsy City by Design: A Theory for Jane Jacobs' Imperfect Cities? \\
\hline $\mathrm{H}$ & Tilaki et al. (2013) & $\begin{array}{l}\text { The influence of landuse planning on the making of the cities physical identity in } \\
\text { Iran: An analytical review and commentary }\end{array}$ \\
\hline $\mathrm{H}$ & Madureira (2015) & Physical planning in place-making through design and image building \\
\hline 1 & Moulay \& Ujang (2016) & $\begin{array}{l}\text { Legibility of neighborhood parks and its impact on social interaction in a planned } \\
\text { residential area }\end{array}$ \\
\hline 1 & Pour Ebrahim (2015) & Sense of Community in New Urbanism Neighbourhoods: A Review \\
\hline $\mathrm{J}$ & Lee (2013) & Perceived neighborhood environment and transit use in low-income populations \\
\hline $\mathrm{K}$ & Shapiro (2016) & The mezzanine \\
\hline $\mathrm{L}$ & Sternberg (2002) & $\begin{array}{l}\text { What makes buildings catalytic? How cultural facilities can be designed to spur } \\
\text { surrounding development }\end{array}$ \\
\hline M & Hall (2008) & $\begin{array}{l}\text { The form-based development plan: Bridging the gap between theory and } \\
\text { practice in urban morphology }\end{array}$ \\
\hline$A$ & Kim et al. (2016) & $\begin{array}{l}\text { An analysis of the relationship between pedestrian traffic volumes and built } \\
\text { environment around metro stations in Seoul }\end{array}$ \\
\hline$A$ & Xinxin \& Jie (2017) & $\begin{array}{l}\text { Study on Urban Design of Luohu Railway Station Area from the Perspective of } \\
\text { Integrated Urban Design. }\end{array}$ \\
\hline$A$ & Vale (2015) & $\begin{array}{l}\text { Transit-oriented development, integration of land use and transport, and } \\
\text { pedestrian accessibility: } \\
\text { Combining node-place model with pedestrian shed ratio to evaluate and classify } \\
\text { station areas in Lisbon }\end{array}$ \\
\hline$A$ & Chow (2014) & $\begin{array}{l}\text { Urban Design, Transport Sustainability and Residents' Perceived Sustainability: A } \\
\text { Case Study of Transit-oriented Development in Hong Kong }\end{array}$ \\
\hline
\end{tabular}




\begin{tabular}{|c|c|c|}
\hline ID & Autor/Ano & Título do artigo \\
\hline A & Payton \& Hawkes (2013) & Designing new transit systems using a transect-based model \\
\hline A & Nikolaeva (2012) & $\begin{array}{l}\text { Designing Public Space for Mobility: Contestation, Negotiation and Experiment at } \\
\text { Amsterdam Airport Schiphol }\end{array}$ \\
\hline A & $\begin{array}{l}\text { Kopic \& Kurtović-Folić } \\
(2011)\end{array}$ & $\begin{array}{l}\text { Principles of the design of urban space that support rail systems from the aspect of } \\
\text { urban elements and elements of the relief }\end{array}$ \\
\hline A & Ha et al. (2011) & $\begin{array}{l}\text { An empirical study on sustainable walkability indices for transit-oriented } \\
\text { development by using the analytic network process approach }\end{array}$ \\
\hline A & Jou (2011) & $\begin{array}{l}\text { Evaluating integration between public transportation and pedestrian-oriented } \\
\text { urban spaces in two main metro stations of Tehran }\end{array}$ \\
\hline A & Olaru et al. (2011) & Residential location and transit-oriented development in a new rail corridor \\
\hline A & Zacharias et al. (2011) & Tokyo Station City: The railway station as urban place \\
\hline A & Sung \& Oh (2011) & $\begin{array}{l}\text { Transit-oriented development in a high-density city: Identifying its association with } \\
\text { transit ridership in Seoul, Korea }\end{array}$ \\
\hline A & Cervero (2007) & $\begin{array}{l}\text { Transit-Oriented Development's Ridership Bonus: A Product of Self-Selection and } \\
\text { Public Policies }\end{array}$ \\
\hline A & Cervero (2009) & $\begin{array}{l}\text { Transport Infrastructure and Global Competitiveness: Balancing Mobility and } \\
\text { Livability }\end{array}$ \\
\hline A & Guttenplan et al. (2003) & $\begin{array}{l}\text { Planning-Level Areawide Multimodal Level-of-Service Analysis: Performance } \\
\text { Measures for Congestion Management }\end{array}$ \\
\hline $\mathrm{B}$ & Littke (2016) & $\begin{array}{l}\text { Revisiting the San Francisco parklets problematizing publicness, parks, and } \\
\text { transferability }\end{array}$ \\
\hline $\mathrm{B}$ & Weinberger (2014) & Three faces of parking: Emerging trends in the U.S \\
\hline$B$ & Mukhija \& Shoup (2006) & Quantity versus quality in off-street parking requirements \\
\hline $\mathrm{B}$ & Shoup (1999) & In lieu of required parking \\
\hline $\mathrm{B}$ & Shoup (1997) & The high cost of free parking \\
\hline C & Grgic et al. (2014) & $\begin{array}{l}\text { Street as a paradigm of pleasant life - influence of the mediaeval city within } \\
\text { diocletian's palace on the new urban typology of modern Split }\end{array}$ \\
\hline $\mathrm{C}$ & Araújo et al. (2011) & $\begin{array}{l}\text { Transporte público coletivo: discutindo acessibilidade, mobilidade e qualidade } \\
\text { de vida }\end{array}$ \\
\hline $\mathrm{D}$ & Wright (2001) & Latin American busways: Moving people rather than cars \\
\hline A & $\begin{array}{l}\text { Santos Nouri \& Costa } \\
(2017)\end{array}$ & $\begin{array}{l}\text { Placemaking and climate change adaptation: new qualitative and quantitative } \\
\text { considerations for the "Place Diagram" }\end{array}$ \\
\hline A & Lin et al. (2017) & $\begin{array}{l}\text { The Impact of Urban Design Descriptors on Outdoor Thermal Environment: A } \\
\text { Literature Review }\end{array}$ \\
\hline A & Ragheb et al. (2016) & $\begin{array}{l}\text { Microclimate and human comfort considerations in planning a historic urban } \\
\text { quarter }\end{array}$ \\
\hline A & $\begin{array}{l}\text { Rodríguez Algeciras et al. } \\
(2015)\end{array}$ & $\begin{array}{l}\text { Human thermal comfort conditions and urban planning in hot-humid climates- } \\
\text { The case of Cuba }\end{array}$ \\
\hline A & Jamei et al. (2015) & $\begin{array}{l}\text { Review on the impact of urban geometry and pedestrian level greening on } \\
\text { outdoor thermal comfort }\end{array}$ \\
\hline A & Djukic et al. (2016) & $\begin{array}{l}\text { Principles of climate sensitive urban design analysis in identification of suitable } \\
\text { urban design proposals. Case study: Central zone of Leskovac competition. }\end{array}$ \\
\hline A & Swapan (2016) & Holistic strategy for urban design: A microclimatic concern to sustainability \\
\hline A & Santos Nouri (2015) & $\begin{array}{l}\text { A framework of thermal sensitive urban design benchmarks: Potentiating the } \\
\text { longevity of Auckland's public realm }\end{array}$ \\
\hline A & Yahia \& Johansson (2014) & $\begin{array}{l}\text { Landscape interventions in improving thermal comfort in the hot dry city of } \\
\text { Damascus, Syria-The example of residential spaces with detached buildings }\end{array}$ \\
\hline A & $\begin{array}{l}\text { Del Castillo Oyarzún \& } \\
\text { Castillo Haeger (2014) }\end{array}$ & $\begin{array}{l}\text { Aproximación bioclimática para el diseño de espacios públicos, análisis inicial en } \\
\text { distintas plazas chilenas }\end{array}$ \\
\hline A & Bajšanski et al. (2015) & $\begin{array}{l}\text { Evaluation and improvement of outdoor thermal comfort in urban areas on } \\
\text { extreme temperature days: Applications of automatic algorithms }\end{array}$ \\
\hline A & Chatterjee (2009) & Smart growth: a solution to climate change? \\
\hline A & Mills (2005) & Progress toward sustainable settlements: a role for urban climatology \\
\hline A & Monteiro et al. (2013) & $\begin{array}{l}\text { A (in)eficácia das políticas europeias e nacionais para prevenir os riscos causados } \\
\text { pelas manifestações de mudança climática nos espaços urbanos }\end{array}$ \\
\hline A & Barros et al. (2010) & O projeto de parque urbano e os riscos da exposição ao calor \\
\hline A & Lenzholzer (2009) & $\begin{array}{l}\text { Engrained experience-a comparison of microclimate perception schemata and } \\
\text { microclimate measurements in Dutch urban squares }\end{array}$ \\
\hline A & Eliasson et al. (2007) & Climate and behaviour in a Nordic city \\
\hline
\end{tabular}




\begin{tabular}{|c|c|c|}
\hline ID & Autor/Ano & Título do artigo \\
\hline B & $\begin{array}{l}\text { Rodríguez-Rojas et al. } \\
\text { (2017) }\end{array}$ & $\begin{array}{l}\text { The paradigm change of urban drainage management from the planning } \\
\text { perspective. A methodological proposal }\end{array}$ \\
\hline $\mathrm{B}$ & Ruban (2016) & Natural-hydrological protection for waterfront territories: "blue-green" initiative \\
\hline $\mathrm{B}$ & Nyka (2016) & Polder and city: sustaining water landscapes on an urban edge. \\
\hline $\mathrm{B}$ & Rodríguez et al. (2014) & Planning criteria for water sensitive urban design \\
\hline $\mathrm{B}$ & Ashley et al. (2013) & Water-sensitive urban design: opportunities for the UK \\
\hline $\mathrm{B}$ & Fryd et al. (2013) & Water sensitive urban design retrofits in Copenhagen - $40 \%$ to the sewer, $60 \%$ to the city \\
\hline B & Nunes et al. (2011) & $\begin{array}{l}\text { Incorporating Water Sensitive Urban Design (WSUD) practices into the planning } \\
\text { context: The conceptual case for lot-scale developments }\end{array}$ \\
\hline B & Urrutiaguer et al. (2010) & $\begin{array}{l}\text { Determining water sensitive urban design project benefits using a multi-criteria } \\
\text { assessment tool. }\end{array}$ \\
\hline C & Taylor \& Hochuli (2015) & $\begin{array}{l}\text { Creating better cities: how biodiversity and ecosystem functioning enhance urban } \\
\text { residents' wellbeing }\end{array}$ \\
\hline C & Bekessy et al. (2012) & Transparent planning for biodiversity and development in the urban fringe \\
\hline $\mathrm{C}$ & Ferguson (2012) & A unified model for integral city design \\
\hline C & Chan \& Lee (2009) & $\begin{array}{l}\text { Design considerations for environmental sustainability in high density } \\
\text { development: A case study of Hong Kong }\end{array}$ \\
\hline C & $\begin{array}{l}\text { Pickett \& Cadenasso } \\
\text { (2007) }\end{array}$ & $\begin{array}{l}\text { Linking Ecological and Built Components of Urban Mosaics: An Open Cycle of } \\
\text { Ecological Design }\end{array}$ \\
\hline $\mathrm{C}$ & Schaefer \& Higgs (2007) & Modern Babylon \\
\hline D & $\begin{array}{l}\text { Yiannakou \& Salata } \\
\text { (2017) }\end{array}$ & $\begin{array}{l}\text { Adaptation to climate change through spatial planning in compact urban areas: } \\
\text { A case study in the City of Thessaloniki }\end{array}$ \\
\hline $\mathrm{D}$ & Siqueira-Gay et al. (2017) & $\begin{array}{l}\text { Vulnerabilidade às ilhas de calor no município de São Paulo: uma abordagem } \\
\text { para a implantação de medidas mitigadoras na gestão urbana }\end{array}$ \\
\hline $\mathrm{D}$ & Kleerekoper et al. (2011) & How to make a city climate-proof, addressing the urban heat island effect \\
\hline $\mathrm{D}$ & Gago et al. (2013) & The city and urban heat islands: A review of strategies to mitigate adverse effects \\
\hline $\mathrm{E}$ & Hamada et al. (2013) & $\begin{array}{l}\text { Impacts of land use and topography on the cooling effect of green areas on } \\
\text { surrounding urban areas }\end{array}$ \\
\hline $\mathrm{E}$ & Sinemillioglv et al. (2010) & $\begin{array}{l}\text { Relationship between green areas and Urban conservation in historical areas and } \\
\text { its reflections: Case of Diyarbakir city, Turkey }\end{array}$ \\
\hline $\mathrm{E}$ & Dagenhart et al. (2006) & Brownfields and urban design: Learning from Atlantic Station \\
\hline $\mathrm{F}$ & Tork et al. (2017) & $\begin{array}{l}\text { Análise da ventilação natural conforme planos diretores: resultados de pesquisa } \\
\text { em Belém, PA }\end{array}$ \\
\hline $\mathrm{F}$ & Yuan et al. (2016) & $\begin{array}{l}\text { A modelling-mapping approach for fine-scale assessment of pedestrian-level } \\
\text { wind in high-density cities }\end{array}$ \\
\hline G & Speranza \& Viader (2015) & High resolution air quality and urban design \\
\hline $\mathrm{H}$ & $\begin{array}{l}\text { Gonçalves \& Meneguetti } \\
\text { (2015) }\end{array}$ & Projeto de arborização como patrimônio da cidade \\
\hline A & Özgür (2013) & $\begin{array}{l}\text { Urban design projects and the planning process: The Kadıköy Old Market Area } \\
\text { Revitalization Project and the Kartal Industrial Area Regeneration Project }\end{array}$ \\
\hline A & Chamberlain (2017) & Zoning at 100 \\
\hline A & Palermo \& Ponzini (2012) & $\begin{array}{l}\text { At the Crossroads between Urban Planning and Urban Design: Critical Lessons } \\
\text { from Three Italian Case Studies }\end{array}$ \\
\hline A & Lind (2007) & Zoning, Urban Form, and Civic Identity: The Future of Pittsburgh's Hillsides Pittsburgh \\
\hline A & Punter (2002) & $\begin{array}{l}\text { Urban Design as Public Policy: Evaluating the Design Dimension of Vancouver's } \\
\text { Planning System }\end{array}$ \\
\hline A & Hall (2000) & A new paradigm for local development plans \\
\hline A & Kamete (1999) & $\begin{array}{l}\text { Restrictive Control of Urban Higg-density Housing in Zimbabwe: Deregulation, } \\
\text { Challenges and Implications for Urban Design }\end{array}$ \\
\hline B & Al-Saffar (2016) & $\begin{array}{l}\text { Toward an integrated sustainable urban design framework in the historic center of } \\
\text { Baghdad }\end{array}$ \\
\hline $\mathrm{B}$ & Zakaria et al. (2012) & Sustainable neighbourhood elements model (SNEs model) \\
\hline $\mathrm{B}$ & Craun (2012) & Refurbishment as a sustainable Urban Design strategy \\
\hline B & $\begin{array}{l}\text { Ercoskun \& Karaaslan } \\
(2011)\end{array}$ & $\begin{array}{l}\text { Next-generation urban design guides for sustainability of small towns: A case study } \\
\text { on Gudul, Turkey }\end{array}$ \\
\hline B & Raeisi et al. (2010) & $\begin{array}{l}\text { Architectural design principles of public spaces based on social sustainability } \\
\text { approach: A case study in Ardabil, Iran }\end{array}$ \\
\hline B & Borst (2008) & $\begin{array}{l}\text { Regenerative french urbanism: Sustainable planning strategies in Lille, Lyon, and } \\
\text { Montpellier }\end{array}$ \\
\hline
\end{tabular}




\begin{tabular}{|c|c|c|}
\hline ID & Autor/Ano & Título do artigo \\
\hline $\mathrm{C}$ & Abe (2003) & $\begin{array}{l}\text { Features of Initial Framework for Urban Regeneration Policies at the Historic Center } \\
\text { of Barcelona }\end{array}$ \\
\hline C & Elinbaum (2014) & $\begin{array}{l}\text { Plan y proyecto territorial en las Comarcas Centrales de Cataluña: Herramientas } \\
\text { de ordenación para una nueva escala de ciudad }\end{array}$ \\
\hline C & Pérez Lancellotti (2014) & $\begin{array}{l}\text { The master plan as a tool for urban design: Potentials and restrictions. The case of } \\
\text { Antofagasta }\end{array}$ \\
\hline $\mathrm{C}$ & Marina \& Velevski (2014) & $\begin{array}{l}\text { Urban Strategies for the Future Development of Turin and Skopje: The Economic } \\
\text { Crisis and the Effectiveness of Urban Design. }\end{array}$ \\
\hline C & Bejleri et al. (2003) & $\begin{array}{l}\text { Florida's Efficient Transportation Decision-Making Process: Laying the Technology } \\
\text { Foundation }\end{array}$ \\
\hline $\mathrm{D}$ & Punter (2007) & $\begin{array}{l}\text { Developing Urban Design as Public Policy: Best Practice Principles for Design } \\
\text { Review and Development Management }\end{array}$ \\
\hline $\mathrm{D}$ & Wey (2011) & $\begin{array}{l}\text { A study of the built environment design elements embedded into the multiple } \\
\text { criteria strategic planning model for an urban renewal }\end{array}$ \\
\hline $\mathrm{D}$ & Gaffikin et al. (2010) & Creating shared public space in the contested city: The role of urban design \\
\hline $\mathrm{D}$ & Lubell (2008) & In a desert city, a skyline grows ever higher. \\
\hline $\mathrm{D}$ & Southworth (2003) & $\begin{array}{l}\text { Urban design in action: The City of Cape Town's Dignified Places Programme - } \\
\text { Implementation of new public spaces towards integration and urban } \\
\text { regeneration in South Africa }\end{array}$ \\
\hline $\mathrm{D}$ & Inam (2002) & Meaningful Urban Design: Teleological/Catalytic/Relevant \\
\hline $\mathrm{E}$ & Raman (2010) & $\begin{array}{l}\text { Designing a liveable compact city physical forms of city and social life in urban } \\
\text { neighbourhoods }\end{array}$ \\
\hline $\mathrm{E}$ & Woodcock et al. (2010) & Modelling the compact city: Capacities and visions for Melbourne \\
\hline $\mathrm{E}$ & Buxton \& Scheurer (2007) & Density and Outer Urban Development in Melbourne \\
\hline $\mathrm{F}$ & Dovey \& Symons (2014) & $\begin{array}{l}\text { Density without intensity and what to do about it: reassembling public/private } \\
\text { interfaces in Melbourne's Southbank hinterland }\end{array}$ \\
\hline $\mathrm{F}$ & Patel (2011) & $\begin{array}{l}\text { Analyzing urban layouts - can high density be achieved with good living } \\
\text { conditions? }\end{array}$ \\
\hline G & Ford (2008) & World Cities and Global Change: Observations on Monumentality in Urban Design \\
\hline $\mathrm{H}$ & Furuseth (1997) & Neotraditional planning: A new strategy for building neighborhoods? \\
\hline \multicolumn{3}{|c|}{ Legenda: } \\
\hline A-M & \multicolumn{2}{|c|}{ Valorização da escala humana nos espaços urbanos. } \\
\hline$A-D$ & \multicolumn{2}{|c|}{ Melhoria na infraestrutura de transporte. } \\
\hline $\mathrm{A}-\mathrm{H}$ & \multicolumn{2}{|c|}{ Busca do equilíbrio ambiental. } \\
\hline $\mathrm{A}-\mathrm{H}$ & fão da escala & umana nos espac \\
\hline
\end{tabular}

